\title{
Visual saliency detection for RGB-D images under a Bayesian framework
}

\author{
Songtao Wang ${ }^{1,2}$ (D), Zhen Zhou ${ }^{1 *}$, Wei Jin ${ }^{2}$ and Hanbing Qu ${ }^{2}$
}

\begin{abstract}
In this paper, we propose a saliency detection model for RGB-D images based on the deep features of RGB images and depth images within a Bayesian framework. By analysing 3D saliency in the case of RGB images and depth images, the class-conditional mutual information is computed for measuring the dependence of deep features extracted using a convolutional neural network; then, the posterior probability of the RGB-D saliency is formulated by applying Bayes' theorem. By assuming that deep features are Gaussian distributions, a discriminative mixed-membership naive Bayes (DMNB) model is used to calculate the final saliency map. The Gaussian distribution parameters can be estimated in the DMNB model by using a variational inference-based expectation maximization algorithm. The experimental results on RGB-D images from the NLPR dataset and NJU-DS400 dataset show that the proposed model performs better than other existing models.
\end{abstract}

Keywords: Bayesian fusion, Deep learning, Generative model, Saliency detection, RGB-D images

\section{Introduction}

Saliency detection is a fundamental problem in computer vision that aims to highlight visually salient regions or objects in an image. Le Callet and Niebur introduced the concepts of overt and covert visual attention and the concepts of bottom-up and top-down processing [1]. Visual attention models have been successfully applied in many domains, including multimedia delivery, visual retargeting, quality assessment of images and videos, medical imaging, and 3D image applications [1]. Today, with the development of 3D display technologies and devices, various applications are emerging for 3D multimedia, such as 3D video retargeting [2], 3D video quality assessment $[3,4]$, and so forth. Overall, the emerging demand for visual attention-based applications for 3D multimedia has increased the need for computational saliency detection models for 3D multimedia content.

Salient object detection has attracted a lot of interest in computer vision [5]. Numerous efforts have been devoted to designing different low-level saliency cues for

\footnotetext{
*Correspondence: zhzh49@126.com

${ }^{1}$ The Higher Educational Key Laboratory for Measuring and Control

Technology and Instrumentations of Heilongjiang Province, Harbin University

of Science and Technology, Harbin, 150080 China

Full list of author information is available at the end of the article
}

2D saliency detection, such as contrast-based features and background priors. Because human attention is preferentially attracted by high-contrast regions with their surroundings, contrast-based features (such as colour, edge orientation, or texture contrast) have a crucial role in deriving salient objects [6]. The background prior leverages the fact that most salient objects are located far from image boundaries [7]. Based on the basic assumption, which non-salient regions (i.e. background) can be explained by the low-rank matrix, salient objects can also be defined as the sparse noises in a certain feature space where the input image is represented as a low-rank matrix [8]. Most existing computational visual saliency models follow a bottom-up framework that generates independent saliency map in each selected visual feature space and combines them in a proper way. To address these problems, Li et al. proposed a saliency map computational model based on tensor analysis [9].

The recently introduced sensing technologies, such as Microsoft Kinect, provide an excellent ability and flexibility to capture RGB-D images. In addition to RGB information, depth has been shown to be one of the practical cues for extracting saliency. Furthermore, Ju et al. proposed a novel saliency method that worked on depth images based on the anisotropic centre-surround difference [10]. In contrast to saliency detection for 2D images, 
the depth factor must be considered when performing saliency detection for RGB-D images. Depth cues provide additional important information about content in the visual field and can therefore also be considered relevant features for saliency detection. With the additional depth information, RGB-D co-saliency detection, which is an emerging and interesting issue in saliency detection, aims to discover the common salient objects in a set of RGB-D images [11]. The stereoscopic content carries important additional binocular cues for enhancing human depth perception $[12,13]$. Therefore, two important challenges when designing 3D saliency models are how to estimate the saliency from depth cues and how to combine the saliency from depth features with those of other 2D low-level features.

In this paper, we propose a new computational saliency detection model based on the deep features of RGB images and depth images within a Bayesian framework. The main contributions of our approach consist of two aspects: (1) to estimate saliency from depth cues, we propose the creation of a depth feature based on a convolutional neural network (CNN) trained by supervision transfer, and (2) by assuming that the deep features of RGB images and depth images are conditionally independent given the classes, the discriminative mixed-membership naive Bayes (DMNB)[14] model is used to calculate the final saliency map by applying Bayes' theorem.

\section{Related work}

In this section, we provide a brief survey and review of RGB-D saliency detection methods. These methods all contain a stage in which 2D saliency features are extracted. However, depending on the way in which they use depth information in terms of developing computational models, these models can be classified into three different categories:

Depth-weighting models This type of model adopts depth information to weight a 2D saliency map to calculate the final saliency map for RGB-D images with feature map fusion [15-18]. Fang et al. proposed a novel 3D saliency detection framework based on colour, luminance, texture, and depth contrast features, and they designed a new fusion method to combine the feature maps to obtain the final saliency map for RGB-D images [15]. In [16], colour contrast features and depth contrast features were calculated to construct an effective multi-feature fusion to generate saliency maps, and multi-scale enhancement was performed on the saliency map to further improve the detection precision, focusing on 3D salient object detection. Ciptadi et al. proposed a novel computational model of visual saliency that incorporates depth information and demonstrated the method by explicitly constructing a 3D layout and shape features from depth measurements [17].
Iatsun et al. proposed a 3D saliency model by relying on 2D saliency features jointly with depth obtained from monocular cues, in which 3D perception is significantly based on monocular cues [18]. The models in this category combine $2 \mathrm{D}$ features with a depth feature to calculate the final saliency map, but they do not include the depth saliency map in their computation processes.

Depth-pooling models This type of model combines depth saliency maps and traditional 2D saliency maps simply to obtain saliency maps for RGB-D images [19-22]. Peng et al. provided a simple fusion framework that combines existing RGB-produced saliency with new depth-induced saliency: the former one is estimated from existing RGB models, whereas the latter one is based on the multi-contextual contrast model [19]. Ren et al. presented a two-stage 3D salient object detection framework, which first integrates the contrast region with the background, depth and orientation priors to achieve a saliency map and then reconstructs the saliency map globally [20]. Xue et al. proposed an effective visual object saliency detection model via RGB and depth cues with mutually guided manifold ranking and obtained the final result by fusing RGB and depth saliency maps [21]. Wang et al. proposed two different ways to integrate depth information in the modelling of $3 \mathrm{D}$ visual attention, where the measures of depth saliency are derived from the eye movement data obtained from an eye tracking experiment using synthetic stimuli [22]. The models in this category rely on the existence of "depth saliency maps". These depth saliency maps are finally combined with $2 \mathrm{D}$ saliency maps using a saliency map pooling strategy to obtain the final 3D saliency map.

Learning-based models Rather than using a depth saliency map directly, this type of model uses machine learning techniques to construct a 3D saliency detection model for RGB-D images based on extracted 2D features and depth features [23-26]. Inspired by the recent success of machine learning techniques in constructing 2D saliency detection models, Fang et al. proposed a learningbased model for RGB-D images using a linear SVM [23]. Zhu et al. proposed a learning-based approach for extracting saliency from RGB-D images, in which discriminative features can be automatically selected by learning several decision trees based on the ground truth, and those features are further utilized to search the saliency regions via the predictions of the trees [24]. Bertasius et al. developed an EgoObject Representation, which encodes these characteristics by incorporating shape, location, size, and depth features from an egocentric RGB-D image, and trained a random forest regressor to predict the saliency of a region using the ground-truth salient object [25]. Qu et al. designed a new $\mathrm{CNN}$ to fuse different low-level 
saliency cues into hierarchical features for automatically detecting salient objects in RGB-D images [26].

Most existing approaches for 3D saliency detection either treat the depth feature as an indicator to weight the RGB saliency map [15-18] or consider the 3D saliency map as the fusion of saliency maps of these low-level features [19-22]. It is not clear how to integrate 2D saliency features with depth-induced saliency feature in a better way, and linearly combining the saliency maps produced by these features cannot guarantee better results. Some other more complex combination algorithms have been proposed. These methods combine the depth-induced saliency map with the 2D saliency map either directly [19] or in a hierarchical way to calculate the final RGBD saliency map [20]. However, because they are restricted by the computed saliency values, these saliency map combination methods are not able to correct incorrectly estimated salient regions. From the above description, the key to $3 \mathrm{D}$ saliency detection models is determining how to integrate the depth cues with traditional 2D low-level features.

In this paper, we focus on how to integrate RGB and the additional depth information for RGB-D saliency detection. This saliency-map-level integration is not optimal because it is restricted by the determined saliency values.
Conversely, we incorporate colour and depth cues at the feature level within a Bayesian framework.

\section{The proposed approach}

In this section, we introduce a method that integrates the colour saliency probability with the depth saliency probability computed from Gaussian distributions based on deep features and yields a prediction of the final 3D saliency map using the DMNB model within a Bayesian framework. The general architecture of the proposed framework is presented in Fig. 1.

First, we train a CNN model for depth images by teaching the network to reproduce the mid-level semantic representation learned from RGB images for which there are paired images. Then, deep features of the RGB and depth images are extracted by a CNN.

Second, the class-conditional mutual information (CMI) is computed to measure the dependence of the deep features of the RGB and depth images; then, the posterior probability of the RGB-D saliency is formulated by applying Bayes' theorem. These two features complement each other in detecting 3D saliency cues from different perspectives and, when combined, yield the final 3D saliency value. By assuming that deep features are Gaussian distributions, the parameters of the Gaussian

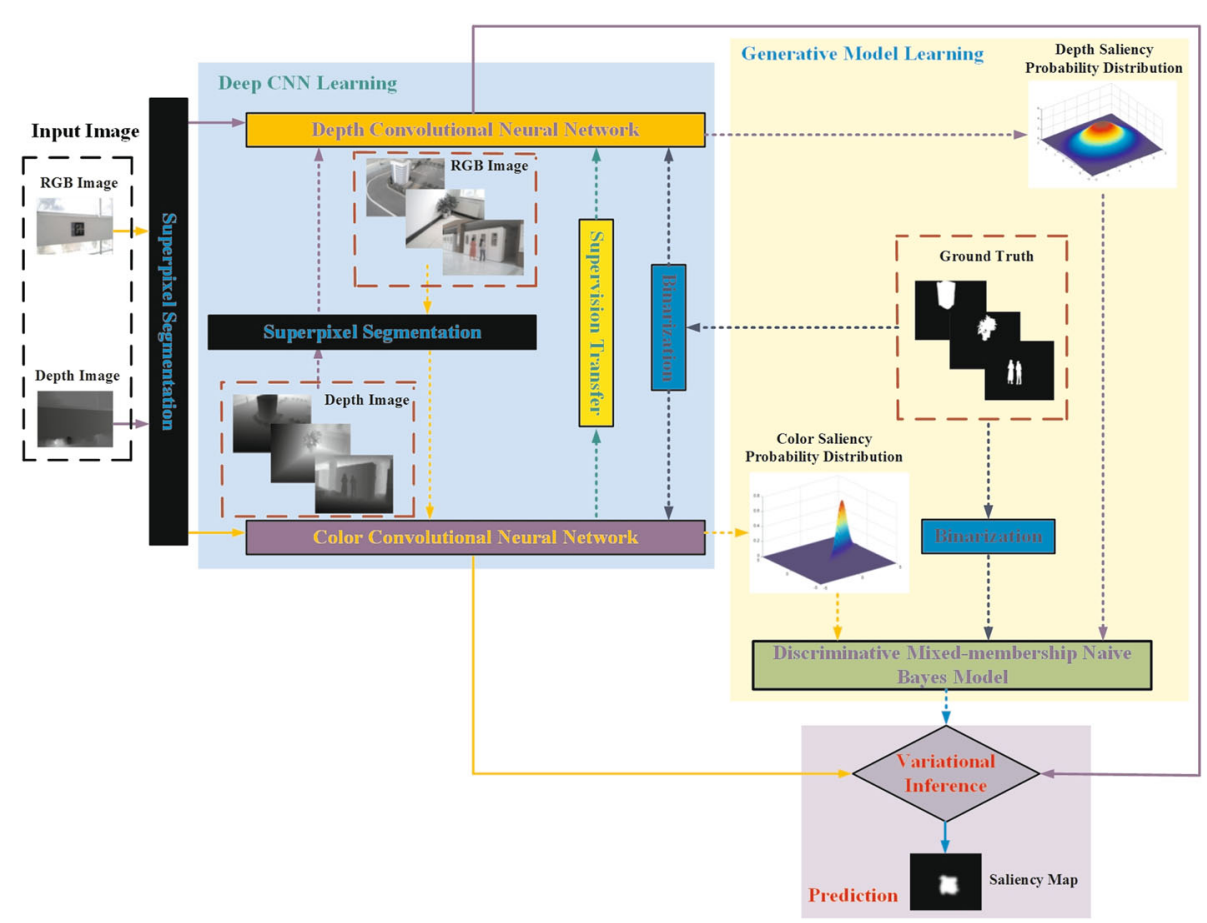

Fig. 1 The flowchart of the proposed model. The framework of our model consists of two stages: the training stage, which includes a depth CNN trained for feature learning and a generative process for saliency, and the testing stage. From a pair of RGB and depth images, our model extracts deep features using a colour CNN and depth CNN, respectively, and performs saliency prediction using the DMNB model [14] within a Bayesian framework. In this work, we perform experiments based on the NLPR dataset in [19] 
distribution can be estimated in the DMNB model using a variational inference-based expectation maximization (EM) algorithm.

\subsection{Feature extraction using CNN}

Most existing saliency detection methods focus on how to design low-level saliency cues or model background priors. Low-level saliency cues alone do not produce good saliency detection results, particularly when salient objects are present in a low-contrast background with confusing visual cues. Objects cannot be classified as salient objects from the low-contrast background either based on low-level saliency cues or background priors, but they are semantically salient in high-level cognition as they are distinct in object categories. Due to its capability of learning high-level semantic features, a $\mathrm{CNN}$ is effective for estimating the saliency maps of images and has been used for saliency detection [27, 28]. A CNN is able to generate representative and discriminative hyperfeatures rather than hand-designing heuristical features for saliency.

To better detect semantically salient objects, it is important to use high-level knowledge on object categories.
We employ deep convolutional neural networks to model the saliency of objects in RGB images and depth images. As shown in Fig. 2, the upper branch of our saliency detection pipeline is a deep $\mathrm{CNN}$ architecture with global context for RGB images, and the lower branch of our saliency detection pipeline is a deep $\mathrm{CNN}$ architecture with global context for depth images. For RGB images, the Clarifai [29] model is adopted as the baseline model, and a task-specific pre-training scheme is designed to make the global-context modelling suitable for saliency detection [27]. We use a CNN similar to the Clarifai model for saliency detection with a pre-training using supervision transfer [30] for limited labelled depth images. The supervision transfer occurs at the penultimate layer of the global context model. Taking the output of the penultimate layer of the two global context models as input, the DMNB model is trained to classify background and saliency, indicating the probabilities of whether a centred superpixel is in the background or belongs to a salient object.

\subsubsection{Deep features of RGB image}

Superpixel segmentation is first performed on RGB-D images [31], and the input of the global-context $\mathrm{CNN}$ is

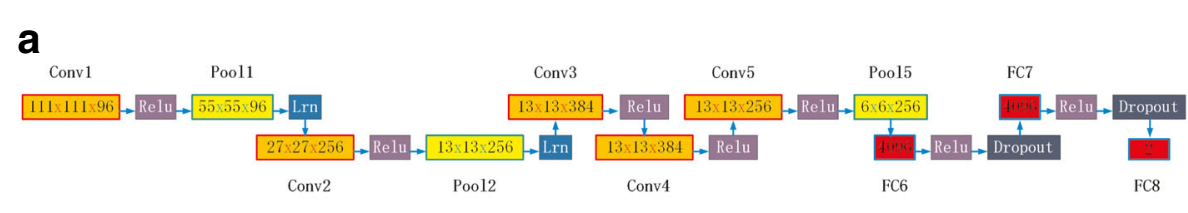

b

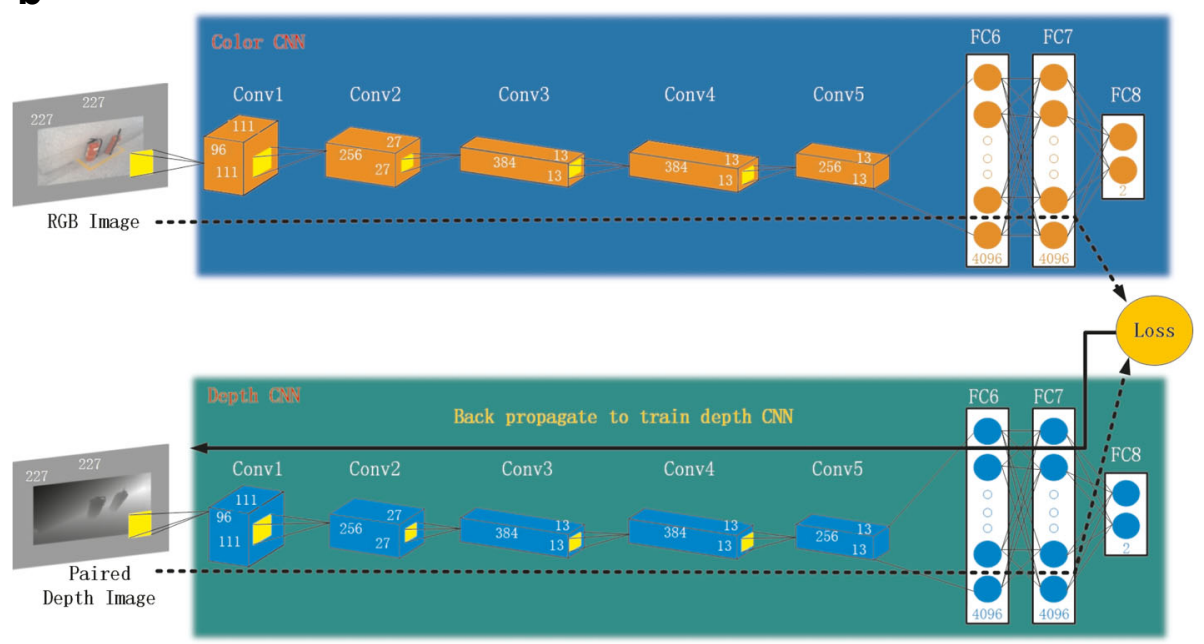

Fig. 2 Architecture for supervision transfer. a The Architecture of Clarifai model, where Relu denotes a rectified linear function relu $(x)=$ max $(x, 0)$, which rectify the feature maps thus ensuring the feature maps are always positive, Irn denotes a local response normalization layer, and Dropout is used in the fully connected layers with a rate of 0.5 to prevent CNN from overfitting. b Upper branch: Deep CNN-based global-context modelling for RGB saliency detection with a superpixel-centred window padded with the mean pixel value of the RGB training dataset. Lower branch: Deep CNN-based global-context modelling for depth saliency detection with a superpixel-centred window padded with the mean pixel value of the depth training dataset. We train a CNN model for depth images by teaching the network to reproduce the mid-level semantic representation learned from RGB images for which there are paired images. The supervision transfer occurs at the penultimate layer of the global context model. For the loss function, we use the $L_{2}$ distance 
a superpixel-centred large context window that includes the full RGB image. Regions that exceed the image boundaries are padded with the mean pixel value of the RGB training dataset. The padded images are then warped to $227 \times 3$ as input, where the three dimensions represent width, height, and number of channels. With this normalization and padding scheme, the superpixel to be classified is always located at the centre of the RGB image, and the spatial distribution of the global context is normalized in this way. Moreover, it ensures that the input covers the entire range of the original RGB image. We refer readers to [27] for further details.

\subsubsection{Deep features of depth image}

We demonstrate how we transfer supervision from RGB images to depth images as obtained from a range sensors, such as the Microsoft Kinect, for the downstream task of saliency detection. We consider the domain of RGB images as $\mathcal{M}_{s}$ for which there is large dataset of labelled images $D_{s}$, and we treat depth images as $\mathcal{M}_{d}$ with limited labelled data $D_{d}$ for which we would like to train a rich representation for saliency detection. We use convolutional neural networks as our layered rich representation. For our layered image representation models, we use $\mathrm{CNNs}$ with the network architecture from the Clarifai model.

We denote the deep features of the RGB image as a corresponding $K$ layered rich representation $\Phi=$ $\left\{\phi_{\mathcal{M}_{s}, D_{s}}^{i}, \forall i \in[1 \cdots K]\right\} . \phi_{\mathcal{M}_{s}, D_{s}}^{i}$ is the $i^{t h}$ layer of the Clarifai model for modality $\mathcal{M}_{s}$ that has been trained on labelled images from dataset $D_{s}$. Now, we want to learn the deep features of depth images from modality $\mathcal{M}_{d}$, for which we do not have access to a large dataset of labelled depth images. We have already hand-designed an appropriate CNN architecture $\Psi=\left\{\psi_{\mathcal{M}_{d}}^{i}, \forall i \in[1 \cdots L]\right\}$ from the Clarifai model. The task is then to effectively learn the parameters associated with various operations in the $\mathrm{CNN}$ architecture without having access to a large set of annotated images for modality $\mathcal{M}_{d}$.

The scheme for training the depth $\mathrm{CNN}$ for depth images of modality $\mathcal{M}_{d}$ is to learn the parameters of CNN $\Psi$ such that feature vectors from $\psi_{D_{d}}^{L}\left(I_{d}\right)$ for image $I_{d}$ match the feature vectors from $\psi_{\mathcal{M}_{s}, D_{s}}^{i^{*}}\left(I_{s}\right)$ for its image pair $I_{s}$ in modality $\mathcal{M}_{s}$ for some chosen and fixed layer $i^{*} \in[1 \cdots K]$. By paired images, we mean a set of images of the same scene in two different modalities. We denote these parameters of CNN $W_{d}^{[1 \cdots L]}=\left\{\boldsymbol{w}_{d}^{i}, \forall i \in[1 \cdots L]\right\}$ to be learned by supervision transfer from layer $i^{*}$ in $\Phi$ of modality $\mathcal{M}_{s}$ to layer $L$ in $\Psi$ of modality $\mathcal{M}_{d}$ :

$$
\min _{W_{d}^{[1 \cdots L]}} \sum_{\left(I_{s}, I_{d}\right) \in U_{s, d}} f\left(\psi_{\mathcal{M}_{d}}^{L}\left(I_{d}\right), \phi_{\mathcal{M}_{s}, D_{s}}^{i^{*}}\left(I_{s}\right)\right)
$$

where $U_{s, d}$ denotes the NLPR dataset, which includes paired images from modalities $\mathcal{M}_{s}$ and $\mathcal{M}_{d}$. For the loss function $f$, we use the $L_{2}$ distance between the feature vectors, $f(\cdot)=\|\cdot\|_{2}^{2}$. Then, the deep features of depth images are extracted by $\mathrm{CNN} \Psi$.

\subsection{Bayesian framework for saliency detection}

Let the binary random variable $z_{s}$ denote whether a point belongs to a salient class. Given the observed deep features of RGB image $\boldsymbol{x}_{c}$ and the deep features of depth image $\boldsymbol{x}_{d}$ of that point, we formulate the saliency detection as a Bayesian inference problem to estimate the posterior probability at each pixel of the RGB-D image:

$$
p\left(\boldsymbol{z}_{s} \mid \boldsymbol{x}_{c}, \boldsymbol{x}_{d}\right)=\frac{p\left(\boldsymbol{z}_{s}, \boldsymbol{x}_{c}, \boldsymbol{x}_{d}\right)}{p\left(\boldsymbol{x}_{c}, \boldsymbol{x}_{d}\right)}
$$

where $p\left(\boldsymbol{z}_{s} \mid \boldsymbol{x}_{c}, \boldsymbol{x}_{d}\right)$ is shorthand for the probability of predicting whether a pixel is salient, $p\left(\boldsymbol{x}_{c}, \boldsymbol{x}_{d}\right)$ is the likelihood of the observed deep features of RGB images and depth images, and $p\left(\boldsymbol{z}_{s}, \boldsymbol{x}_{c}, \boldsymbol{x}_{d}\right)$ is the joint probability of the latent class and observed features, defined as $p\left(\boldsymbol{z}_{s}, \boldsymbol{x}_{c}, \boldsymbol{x}_{d}\right)=p\left(\boldsymbol{z}_{s}\right) p\left(\boldsymbol{x}_{c}, \boldsymbol{x}_{d} \mid \boldsymbol{z}_{s}\right)$.

In this paper, the class-conditional mutual information (CMI) is used as a measure of the dependence between two features $\boldsymbol{x}_{c}$ and $\boldsymbol{x}_{d}$, which can be defined as $I\left(\boldsymbol{x}_{c}, \boldsymbol{x}_{d} \mid \boldsymbol{z}_{s}\right)=H\left(\boldsymbol{x}_{c} \mid \boldsymbol{z}_{s}\right)+H\left(\boldsymbol{x}_{d} \mid \boldsymbol{z}_{s}\right)-H\left(\boldsymbol{x}_{c}, \boldsymbol{x}_{d} \mid \boldsymbol{z}_{s}\right)$, where $H\left(\boldsymbol{x}_{c} \mid \boldsymbol{z}_{s}\right)$ is the class-conditional entropy of $\boldsymbol{x}_{c}$, defined as $-\sum_{i} p\left(\boldsymbol{z}_{s}=i\right) \sum_{\boldsymbol{x}_{c}} p\left(\boldsymbol{x}_{c} \mid \boldsymbol{z}_{s}=i\right) \log p\left(\boldsymbol{x}_{c} \mid \boldsymbol{z}_{s}=i\right)$. Mutual information is zero when $\boldsymbol{x}_{c}$ and $\boldsymbol{x}_{d}$ are mutually independent given class $z_{s}$ and increases with increasing level of dependence, reaching the maximum when one feature is a deterministic function of the other. Indeed, the independence assumption becomes more accurate with decreasing entropy, which yields an asymptotically optimal performance of the naive Bayes classifier [32].

The visual result for class-conditional mutual information between the deep features of RGB images and depth images on the NLPR dataset is shown in Fig. 5. We employ a CMI threshold $\tau$ to discover feature dependencies. For CMI between the deep features of RGB images and depth images less than $\tau$, we assume that $\boldsymbol{x}_{c}$ and $\boldsymbol{x}_{d}$ are conditionally independent given the classes $\boldsymbol{z}_{s}$, that is, $p\left(\boldsymbol{x}_{c}, \boldsymbol{x}_{d} \mid \boldsymbol{z}_{s}\right)=p\left(\boldsymbol{x}_{c} \mid \boldsymbol{z}_{s}\right) p\left(\boldsymbol{x}_{d} \mid \boldsymbol{z}_{s}\right)$. This entails the assumption that the distribution of the deep features of RGB images does not change with the deep features of depth images. Thus, the pixel-wise saliency of the likelihood is given by $p\left(\boldsymbol{z}_{s} \mid \boldsymbol{x}_{c}, \boldsymbol{x}_{d}\right) \propto p\left(\boldsymbol{z}_{s}\right) p\left(\boldsymbol{x}_{c} \mid \boldsymbol{z}_{s}\right) p\left(\boldsymbol{x}_{d} \mid \boldsymbol{z}_{s}\right)$.

\subsection{Generative model for saliency estimation}

Given the graphical model of DMNB for saliency detection shown in Fig. 3, the generative process for $\left\{x_{1: N}, y\right\}$ following the DMNB model can be described as follows (Algorithm 1), where $\operatorname{Dir}()$ is shorthand for a Dirichlet distribution, Mult() is shorthand for a multinomial distribution, $\boldsymbol{x}_{1: N}=\left(\boldsymbol{x}_{c}, \boldsymbol{x}_{d}\right), \boldsymbol{z}_{1: N}=\boldsymbol{z}_{s}=\left(\boldsymbol{z}_{c}, \boldsymbol{z}_{d}\right), N$ is 


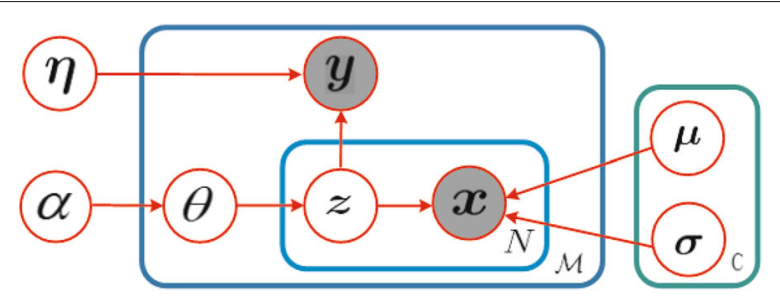

Fig. 3 Graphical models of DMNB for saliency estimation. $\boldsymbol{y}$ and $\boldsymbol{x}$ are the corresponding observed states, and $z$ is the hidden variable, where each feature $\boldsymbol{x}_{j}$ is assumed to have been generated from one of C Gaussian distributions with a mean of $\left\{\mu_{j k},[j]_{1}^{N}\right\}$ and a variance of $\left\{\sigma_{j k}^{2}[j]_{1}^{N}\right\}$, and $\boldsymbol{y}$ is either 0 or 1 , indicating whether the pixel is salient

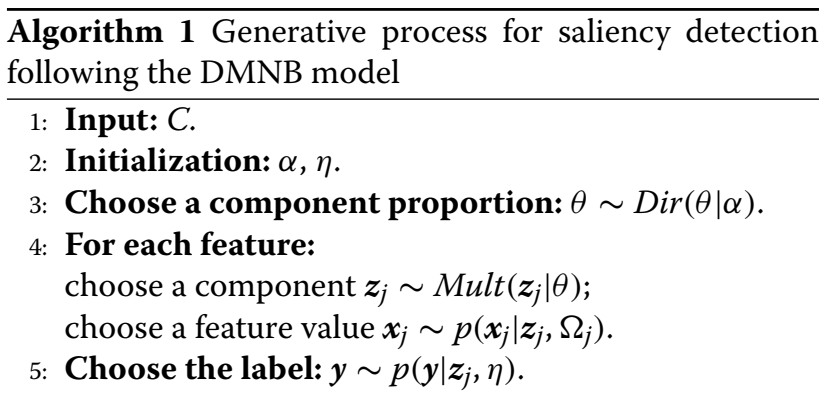

the number of features, and $y$ is the label that indicates whether the pixel is salient.

In this work, the deep features of both RGB and depth images are assumed to have been generated from a Gaussian distribution with a mean of $\left\{\mu_{j k},[j]_{1}^{N}\right\}$ and a variance of $\left\{\sigma_{j k}^{2},[j]_{1}^{N}\right\}$. The marginal distribution of $\left(\boldsymbol{x}_{1: N}, \boldsymbol{y}\right)$ is

$p\left(\boldsymbol{x}_{1: N}, \boldsymbol{y} \mid \alpha, \Omega, \eta\right)=\int p(\theta \mid \alpha)\left(\prod_{j=1}^{N} \sum_{z_{j}} p\left(\boldsymbol{z}_{j} \mid \theta\right) p\left(\boldsymbol{x}_{j} \mid \boldsymbol{z}_{j}, \Omega_{j}\right) p\left(\boldsymbol{y} \mid \boldsymbol{z}_{j}, \eta\right)\right) d \theta$

where $\theta$ is the prior distribution over $C$ components, $\Omega=$ $\left\{\left(\mu_{j k}, \sigma_{j k}^{2}\right),[j]_{1}^{N},[k]_{1}^{C}\right\}$ are the parameters for the distributions of $N$ features, and $p\left(\boldsymbol{x}_{j} \mid \boldsymbol{z}_{j}, \Omega_{j}\right) \triangleq \mathcal{N}\left(\boldsymbol{x}_{j} \mid \mu_{j k}, \sigma_{j k}^{2}\right)$. In two-class classification, $\boldsymbol{y}$ is either 0 or 1 generated from $\operatorname{Bern}(\boldsymbol{y} \mid \eta)$. Because the DMNB model assumes a generative process for both the labels and features, we use both $\mathcal{X}=\left\{\left(\boldsymbol{x}_{i j}\right),[i]_{1}^{\mathcal{M}},[j]_{1}^{N}\right\}$ and $\mathcal{Y}=\left\{\boldsymbol{y}_{i},[i]_{1}^{\mathcal{M}}\right\}$ as a collection of $\mathcal{M}$ superpixels in trained images from the generative process to estimate the parameters of the DMNB model such that the likelihood of observing $(\mathcal{X}, \mathcal{Y})$ is maximized. In practice, we may find a proper $C$ using the Dirichlet process mixture model (DPMM)[33]. The DPMM thus provides a nonparametric prior for the parameters of a mixture model that allows the number of mixture components to increase as the training set increases, as shown in Fig. 6.
Due to the latent variables, the computation of the likelihood in Eq. 3 is intractable. In this paper, we use a variational inference method, which alternates between obtaining a tractable lower bound to the true log-likelihood and choosing the model parameters to maximize the lower bound. By directly applying Jensen's inequality [14], the lower bound to $\log p\left(\boldsymbol{y}, \boldsymbol{x}_{1: N} \mid \alpha, \Omega, \eta\right)$ is given by

$$
\begin{aligned}
\log p\left(\boldsymbol{y}, \boldsymbol{x}_{1: N} \mid \alpha, \Omega, \eta\right) & \geq \boldsymbol{E}_{q}\left(\log p\left(\boldsymbol{y}, \boldsymbol{x}_{1: N}, \boldsymbol{z}_{1: N} \mid \alpha, \Omega, \eta\right)\right) \\
& +\boldsymbol{H}\left(q\left(\boldsymbol{z}_{1: N}, \theta \mid \gamma, \boldsymbol{\phi}\right)\right)
\end{aligned}
$$

Noting that $\boldsymbol{x}_{1: N}$ and $\boldsymbol{y}$ are conditionally independent given $z_{1: N}$, we use a variational distribution:

$$
q\left(z_{1: N}, \theta \mid \gamma, \phi\right)=q(\theta \mid \gamma) \prod_{j=1}^{N} q\left(z_{j} \mid \phi\right)
$$

where $q(\theta, \gamma)$ is a $C$-dimensional Dirichlet distribution for $\theta$ and $q\left(z_{j} \mid \phi\right)$ is a discrete distribution for $z_{j}$. We use $\mathcal{L}$ to denote the lower bound:

$$
\begin{aligned}
\mathcal{L}= & \boldsymbol{E}_{q}[\log p(\theta \mid \alpha)]+\boldsymbol{E}_{q}\left[\log p\left(\boldsymbol{z}_{1: N} \mid \theta\right)\right] \\
& +\boldsymbol{E}_{q}\left[\log p\left(\boldsymbol{x}_{1: N} \mid \boldsymbol{z}_{1: N}, \gamma\right)\right] \\
& -\boldsymbol{E}_{q}[\log q(\theta)]-\boldsymbol{E}_{q}\left[\log q\left(\boldsymbol{z}_{1: N}\right)\right]+\boldsymbol{E}_{q}\left[\log p\left(\boldsymbol{y} \mid \boldsymbol{z}_{1: N}, \eta\right)\right]
\end{aligned}
$$

where $\boldsymbol{E}_{q}\left[\log p\left(\boldsymbol{y} \mid \boldsymbol{z}_{1: N}, \eta\right)\right] \geq \quad \sum_{k=1}^{C} \phi_{k}\left(\eta_{k} \boldsymbol{y}-\frac{e^{\eta_{k}}}{\xi}\right)-$ $\left(\frac{1}{\xi}+\log \xi\right)$ and $\xi>0$ is a newly introduced variational parameter. Maximizing the lower-bound function $\mathcal{L}\left(\gamma_{k}, \phi_{k}, \xi ; \alpha, \Omega, \eta\right)$ with respect to the variational parameters yields updated equations for $\gamma_{k}, \phi_{k}$ and $\xi$ as follows:

$$
\phi_{k} \propto e^{\left(\Psi\left(\gamma_{k}\right)-\Psi\left(\sum_{l=1}^{C} \gamma_{l}\right)+\frac{1}{N}\left(\eta_{k} y_{i}-\frac{e^{\eta} k}{\xi}-\sum_{j=1}^{N} \frac{\left(x_{i j}-\mu_{j k}\right)^{2}}{2 \sigma_{j k}^{2}}\right)\right)}
$$

$$
\begin{aligned}
& \gamma_{k}=\alpha+N \phi_{k} \\
& \xi=1+\sum_{k=1}^{C} \phi_{k} e^{\eta_{k}}
\end{aligned}
$$

The variational parameters $\left(\gamma^{*}, \phi^{*}, \xi^{*}\right)$ from the inference step provide the optimal lower bound to the loglikelihood of $\left(\boldsymbol{x}_{i}, \boldsymbol{y}_{i}\right)$, and maximizing the aggregate lower bound $\sum_{i=1}^{\mathcal{M}} \mathcal{L}\left(\gamma^{*}, \phi^{*}, \xi^{*}, \alpha, \Omega, \eta\right)$ over all data points with respect to $\alpha, \Omega$ and $\eta$, respectively, yields the estimated parameters. For $\mu, \sigma$ and $\eta$, we have $\mu_{j k}=\frac{\sum_{i=1}^{\mathcal{M}} \phi_{i k} x_{i j}}{\sum_{i=1}^{\mathcal{M}} \phi_{i k}}$, $\sigma_{j k}=\frac{\sum_{i=1}^{\mathcal{M}} \phi_{i k}\left(\boldsymbol{x}_{i j}-\mu_{j k}\right)^{2}}{\sum_{i=1}^{\mathcal{M}} \phi_{i k}}$, and $\eta_{k}=\log \left(\frac{\sum_{i=1}^{\mathcal{M}} \phi_{i k} \boldsymbol{y}_{i}}{\sum_{i=1}^{\mathcal{M}} \frac{\phi_{i k}}{\xi_{i}}}\right)$.

Based on the variational inference and parameter estimation updates, it is straightforward to construct a variational EM algorithm to estimate $(\alpha, \Omega, \eta)$. Starting with 


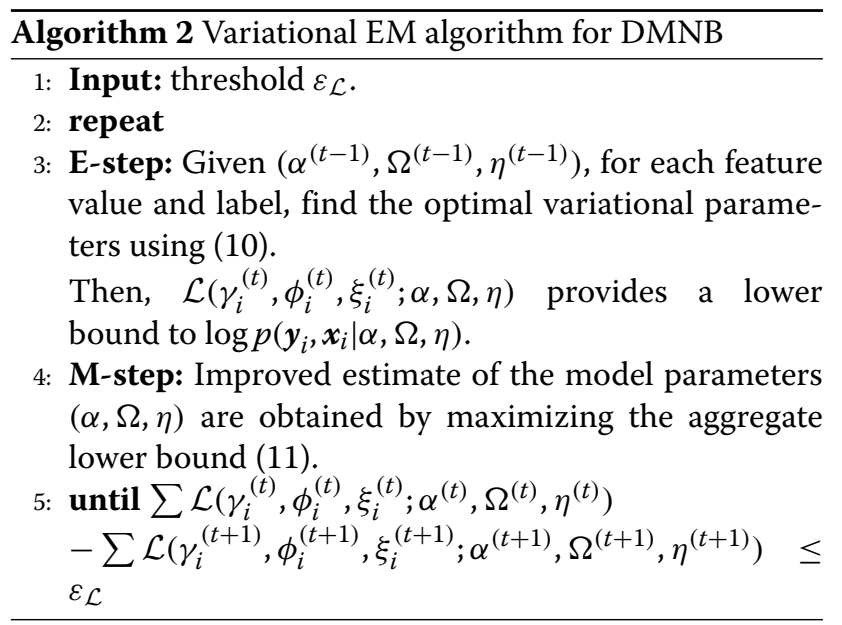

an initial guess $\left(\alpha^{0}, \Omega^{0}, \eta^{0}\right)$, the variational EM algorithm alternates between two steps, as follows (Algorithm 2).

$\left(\gamma_{i}^{(t)}, \phi_{i}^{(t)}, \xi_{i}^{(t)}\right)=\underset{\gamma_{i}, \phi_{i}, \xi_{i}}{\arg \max _{i}} \mathcal{L}\left(\gamma_{i}, \phi_{i}, \xi_{i} ; \alpha^{(t-1)}, \Omega^{(t-1)}, \eta^{(t-1)}\right)$

$\left(\alpha^{(t)}, \Omega^{(t)}, \eta^{(t)}\right)=\arg \max _{(\alpha, \Omega, \eta)} \sum_{i=1}^{\mathcal{M}} \mathcal{L}\left(\gamma_{i}^{(t)}, \phi_{i}^{(t)}, \xi_{i}^{(t)} ; \alpha, \Omega, \eta\right)$

After obtaining the DMNB model parameters from the EM algorithm, we can use $\eta$ to perform saliency prediction. Given the feature $\boldsymbol{x}_{1: N}$, we have

$\boldsymbol{E}\left[\log p\left(\boldsymbol{y} \mid \boldsymbol{x}_{1: N}, \alpha, \Omega, \eta\right)\right]=\left\{\begin{array}{crl}\eta^{T} \boldsymbol{E}[\overline{\boldsymbol{z}}]-\boldsymbol{E}\left[\log \left(1+e^{\eta^{T} \bar{z}}\right)\right] & \boldsymbol{y}=1 \\ 0-\boldsymbol{E}\left[\log \left(1+e^{\eta^{T}}\right)\right] & \boldsymbol{y}=0\end{array}\right.$

where $\bar{z}$ is an average of $z_{1: N}$ over all of the observed features. The computation for $\boldsymbol{E}[\overline{\boldsymbol{z}}]$ is intractable; therefore, we again introduce the distribution $q\left(\boldsymbol{z}_{1: N}, \theta\right)$ and calculate $\boldsymbol{E}_{q}[\overline{\boldsymbol{z}}]$ as an approximation of $\boldsymbol{E}[\overline{\boldsymbol{z}}]$. In particular, $\boldsymbol{E}_{q}[\overline{\boldsymbol{z}}]=\phi$; therefore, we only need to compare $\eta^{T} \phi$ with 0 .

\subsection{Experimental evaluation}

\subsection{Generative model for saliency estimation}

\subsubsection{Evaluation datasets}

In this section, we conduct some experiments to demonstrate the performance of our method. We use the NLPR dataset ${ }^{1}$ and NJU-DS400 datase ${ }^{2}$ to evaluate the performance of the proposed model, as shown in Table 1. The NLPR dataset [19] includes 1000 images of diverse scenes in real 3D environments, where the ground-truth was obtained by requiring five participants to select regions where objects are present, i.e. the salient regions were marked by hand. The NJU-DS400 dataset [10] includes
Table 1 Comparison of the benchmark and existing 3D saliency detection datasets

\begin{tabular}{lllll}
\hline Name & Size & Object no. & Scene types & Centre bias \\
\hline NLPR dataset & 1000 & one (mostly) & 11 & Yes \\
$\begin{array}{l}\text { NJU-DS400 } \\
\text { dataset }\end{array}$ & 400 & one (mostly) & $>10$ & Yes \\
\hline
\end{tabular}

400 images of different scenes, where the ground-truth was obtained by four volunteers labelling the salient object masks.

We will analyse the 3D saliency situation in RGB-D images based on human judgement. In terms of the NLPR dataset [19], the 3D saliency is decided jointly by RGB images and depth images, as shown in Fig. 4. For each selected image pair from NLPR dataset, three participants are asked to draw a rectangle according to their first glance at the the most attention-grabbing region in RGB image and depth image, respectively. The 3D saliency situation is determined by thresholding the overlap ratio between the rectangle and the corresponding ground truth salient object mask. We use the Intersection over Union (IOU) to measure the match between bounding boxes and the ground truth, respectively. The IOU threshold is set at 0.5 . The 3D saliency situation in RGB-D images follows three conditions:

Colour-depth saliency, in which both IOU values of RGB images and depth images are more than the IOU threshold, defined as $\mathcal{D}^{b}=\left\{\mathcal{I}_{c}^{b}, \mathcal{I}_{d}^{b}\right\}$, where $\mathcal{I}_{c}^{b}$ and $\mathcal{I}_{d}^{b}$ denote RGB images and depth images, respectively.

Colour saliency, in which only IOU values of RGB images are more than the IOU threshold and IOU values of depth images are less than the IOU threshold, defined as $\mathcal{D}^{c}=\left\{\mathcal{I}_{c}^{c}, \mathcal{I}_{d}^{c}\right\}$, where $\mathcal{I}_{c}^{c}$ and $\mathcal{I}_{d}^{c}$ denote RGB images and depth images, respectively.

Depth saliency, in which only IOU values of depth images are more than the IOU threshold and IOU values of RGB images are less than the IOU threshold, defined as $\mathcal{D}^{d}=\left\{\mathcal{I}_{c}^{d}, \mathcal{I}_{d}^{d}\right\}$, where $\mathcal{I}_{c}^{d}$ and $\mathcal{I}_{d}^{d}$ denote RGB images and depth images, respectively.

We removed RGB-D image pairs with severely overlapping salient objects and this leaves us with 992 images out of 1000 images from NLPR dataset. The image proportion of the three conditions about 3D saliency in RGB-D images is shown in Table 2. In the NLPR RGB$\mathrm{D}$ dataset, most of the regions are $3 \mathrm{D}$ salient regions in the RGB images and depth images, namely, the colourdepth saliency ratio reaches $76.7 \%$, which is much higher than the colour saliency situation and the depth saliency situation. These split datasets are used for training and evaluation. 


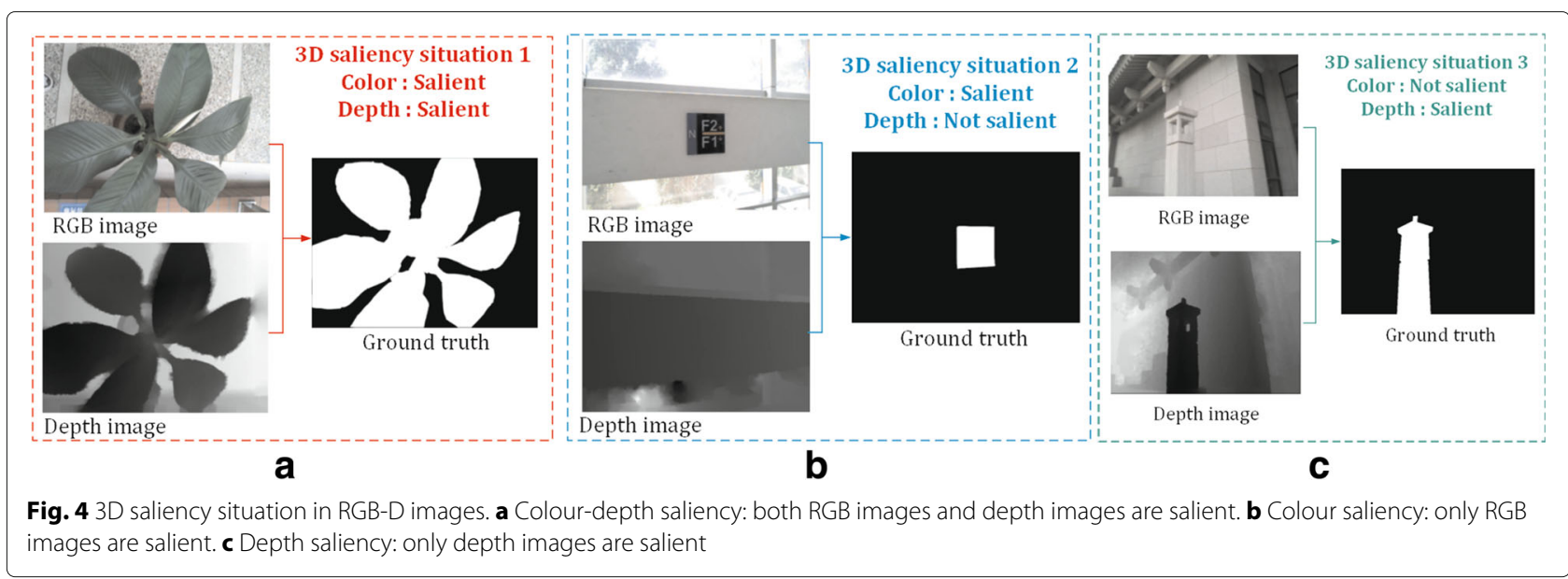

\subsubsection{Evaluation metrics}

There are currently no specific and standardized measures for computing the similarity between the fixation density maps and saliency maps created using computational models in 3D situations. Nevertheless, there is a range of different measures that are widely used to perform comparisons of saliency maps for $2 \mathrm{D}$ content. We introduce two types of measures to evaluate algorithm performance on the benchmark. The first one is the gold standard: F-measure. The second is the precision-recall (PR) curve. A continuous saliency map can be converted into a binary mask using a threshold, resulting in a pair of precision and recall values when the binary mask is compared against the ground truth. A PR curve is then obtained by varying the threshold from 0 to 1 . The PR curve indicates the mean precision and recall of the saliency map at various thresholds.

\subsubsection{Implementation details}

We follow the default setup of the MC procedure from [27] for training the depth CNN using the caffe CNN library [34]. For training the depth $\mathrm{CNN}$ using supervision transfer, we copy the weights from the RGB CNN [27] that was pre-trained on ImageNet Large-Scale Visual Recognition Challenge (ILSVRC) 2014 [35] and fine-tuned for saliency detection on the MSRA10K dataset [36] to initialize this network, base the learning rate at 0.001 and step it down by a factor of 10 every 1000 iterations, except that we fine-tune all the layers. We randomly select 600 depth images $\mathcal{I}_{d}^{b}$ for training and 100 for validation from $\mathcal{D}^{b}$. From each depth image, we select an average 200 of superpixels, and in total, approximately 120 thousand input windows for training and 20 thousand for validation are

Table 2 3D saliency situation in terms of the NLPR dataset

\begin{tabular}{llll}
\hline Dataset & Colour-depth saliency & Colour saliency & Depth saliency \\
\hline NLPR & $76.7 \%$ & $20.8 \%$ & $2.5 \%$ \\
\hline
\end{tabular}

generated. We label a patch as salient if $50 \%$ of the pixels in this patch are salient; otherwise, it is labelled as non-salient. Training of the depth CNN for 10 thousand iterations costs $60 \mathrm{~h}$ without a GPU.

\subsubsection{Parameter settings}

A summary of the parameters in this paper is shown in Table 3. To evaluate the quality of the proposed approach, we divided the datasets into two subsets according to their CMI values, and we kept $20 \%$ of the data for testing purposes and trained on the remaining $80 \%$ whose CMI values are less than the CMI threshold $\tau$. As shown in Fig. 5, we compute the CMI for all of the RGB-D images, and the parameter $\tau$ is set to 0.2 , which is a heuristically determined value.

We initialize the model parameters using all data points and their labels in the training set in Algorithm 1. In particular, we use the mean and standard deviation of the data points in each class to initialize $\Omega$ and the ratio of data points in different classes to initialize $\alpha_{i}$.

\subsubsection{The effect of the parameters}

The parameter $C$ in Algorithm 1 is set according to the training set based on DPMM, as shown in Fig. 6. The appropriate number of mixture components to use in the DMNB model for saliency estimation is generally unknown, and DPMM provides an attractive alternative

Table 3 The parameters and their settings in this paper

\begin{tabular}{lll}
\hline Name & Range & Description \\
\hline$\tau$ & $(0,1)$ & A CMI threshold \\
$\alpha$ & $(0,40]$ & The parameter of a Dirichlet distribution \\
$\theta$ & $(0,1)$ & The parameter of a multinomial distribution \\
$\eta$ & $(-2.0,2.0)$ & The parameter of a Bernoulli distribution \\
$\Omega$ & $((0,1),(0,0.2))$ & The parameter of a Gaussian distribution \\
$C$ & $>2$ & The number of components of DMNB
\end{tabular}




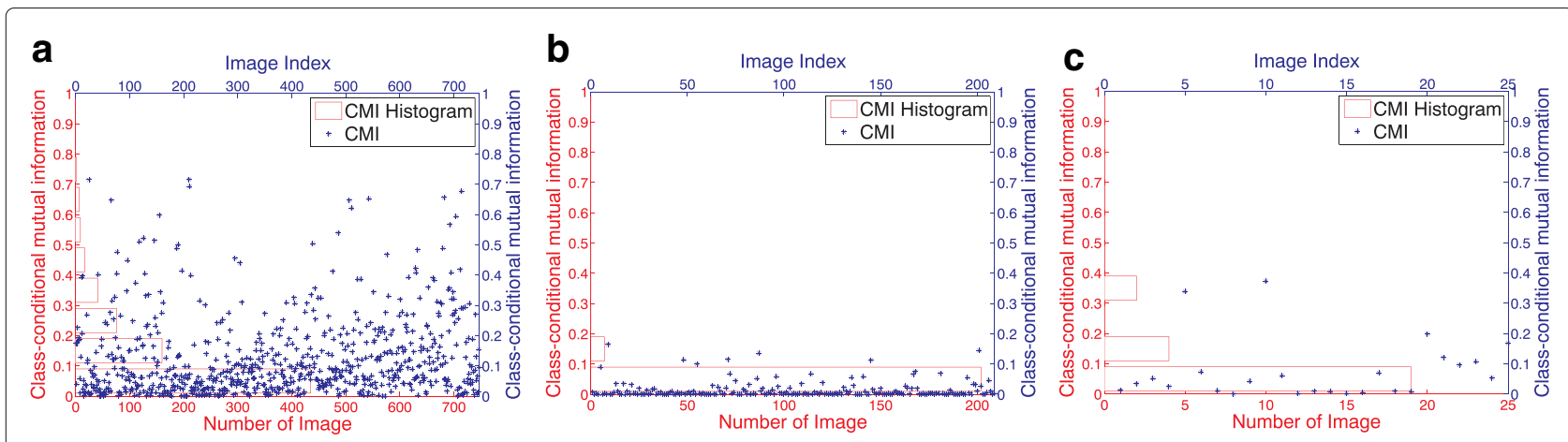

Fig. 5 Visual result for class-conditional mutual information between deep features of RGB images and depth images on the NLPR dataset, where blue star denotes the CMI value and red rectangle denotes the CMI histogram. The 3D saliency situation in RGB-D images is defined as colour-depth saliency, colour saliency, and depth saliency. a Colour-depth saliency. b Colour saliency. c Depth saliency

to current methods. In practice, we find the initial number of components $C$ using the DPMM based on $90 \%$ of the training set, and then we perform a cross validation with a range of $C$ by holding out $10 \%$ of the training data as the validation data.

We use 10 -fold cross-validation with the parameter $C$ for DMNB models. In a 10-fold cross-validation, we divide the dataset evenly into 10 parts, one of which is selected as the validation set, and the remaining 9 parts are used as the training set. The process is repeated 10 times, with each part used once as the validation set. We use perplexity as the measurement for comparison. The generative models are capable of assigning a $\log$-likelihood $\log p\left(\boldsymbol{x}_{i}\right)$ to each observed data point $\boldsymbol{x}_{i}$. Based on the log-likelihood scores, we compute the perplexity of the entire dataset as perplexity $=\exp \left(-\sum_{i=1}^{M} \frac{\log p\left(\boldsymbol{x}_{i}\right)}{M}\right)$, where $M$ is the number of data points. The perplexity is a monotonically decreasing function of the log-likelihood, implying that a lower perplexity is better (particularly on the test set) since the model can explain the data better. We calculate the perplexity for results on the validation set and training set, as shown in Fig. 7. Finally, for all the experiments described below, the parameter $C$ was fixed at 24 , and no user fine-tuning was performed.

\subsubsection{Compared methods}

Let us compare our saliency model (BFSD) with a number of existing state-of-the-art methods, including graphbased manifold ranking (GMR)[7]; multi-context deep learning (MC)[27]; multiscale deep CNN (MDF)[28]; anisotropic centre-surround difference (ACSD)[10]; saliency detection at low-level, mid-level, and high-level stages (LMH) [19]; and exploiting global priors (GP)[20], among which GMR, MC and MDF are developed for RGB images, LMH and GP for RGB-D images, and ACSD for depth images. All of the results are produced using the public codes that are offered by the authors of the previously mentioned literature reports.
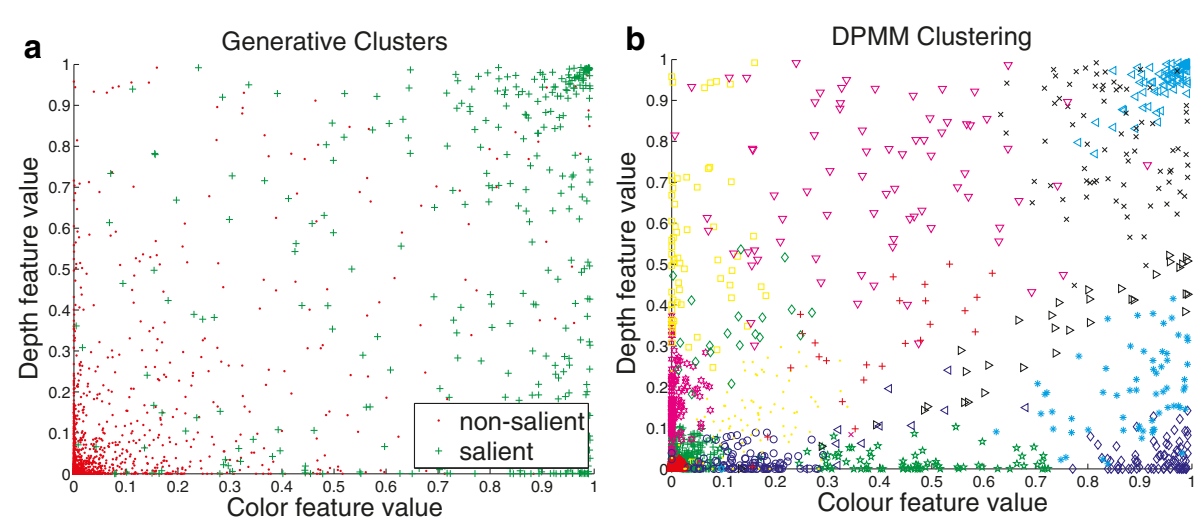

Fig. 6 Visual result for the number of components $C$ in the DMNB model: generative clusters vs DPMM clustering. a Generative clusters for NLPR image datasets, where green and red denote the distributions of salient and non-salient features, respectively. b DPMM clustering for NLPR image datasets, where the number of colours and shapes of the points denote the number of components $C$. The appropriate number of mixture components to use in the DMNB model for saliency estimation is generally unknown, and DPMM provides an attractive alternative to current methods. We find that $C=24$ using DPMM on the NLPR dataset 


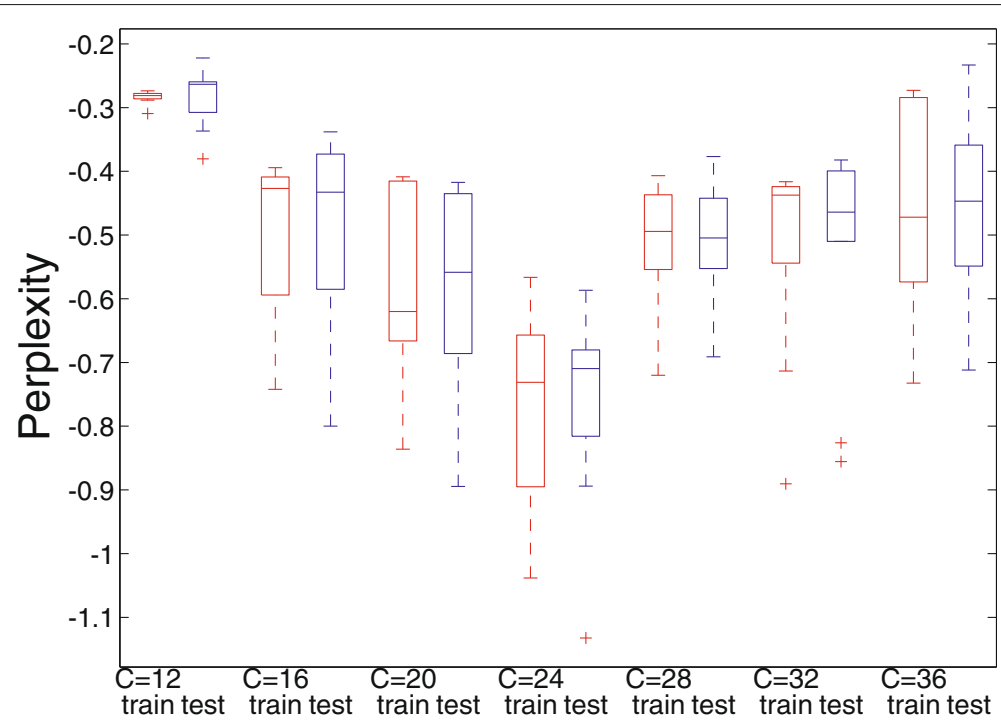

Fig. 7 Cross validation. We use 10-fold cross-validation with the parameter C for DMNB models. The C found using DPMM was adjusted over a wide range in a 10-fold cross-validation

\subsection{Qualitative experiment}

\subsubsection{Colour-depth saliency}

In this case, both RGB images and depth images are salient. The comparison of the state-of-the-art approaches is presented in Fig. 8. As shown in the first and seventh rows of Fig. 8, the salient object has a high colour contrast with the background; thus, RGB saliency methods are able to correctly detect salient objects. GMR fails to detect many pixels on the prominent objects because it does not define the pseudo-background accurately, e.g. the third

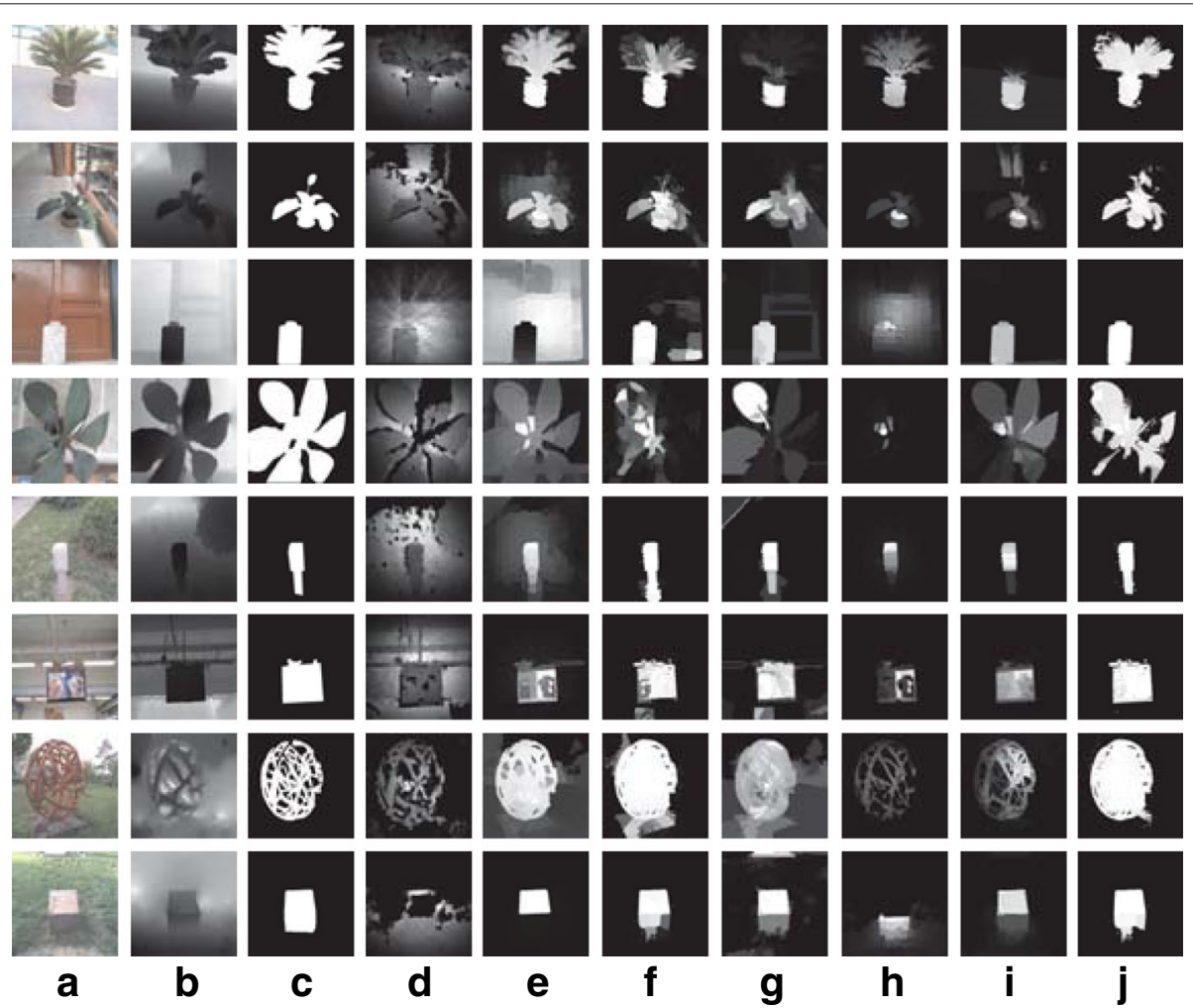

Fig. 8 Visual comparison of the saliency detection in the colour-depth saliency situation in terms of the NLPR dataset. a RGB.b Depth. c Ground truth. d ACSD. e GMR. f MC. g MDF. h LMH. i GP. jBFSD 
row in Fig. 8. As shown, the proposed method can accurately locate the salient objects and produce nearly equal saliency values for the pixels within the target objects.

\subsubsection{Colour saliency}

In this case, only RGB images are salient. The comparison of the state-of-the-art approaches is presented in Fig. 9. ACSD works on depth images on the assumption that salient objects tend to stand out from the surrounding background, which takes relative depth into consideration. ACSD performs worse when the salient object lies in the same plane as the background, e.g. the third row in Fig. 9. It is challenging because most of the salient objects share similar depth as the background. Consequently, depth saliency methods perform relatively worse than RGB saliency methods in terms of precision. Ren et al. proposed two priors, which are the normalized depth prior and the global-context surface orientation prior [20]. Because their approach uses the two priors, it has problems when such priors are invalid, e.g. the first row in Fig. 9. Figure 9 shows that the proposed method consistently outperforms all the other saliency methods.

\subsubsection{Depth saliency}

In this case, only depth images are salient. The comparison of the state-of-the-art approaches is presented in Fig. 10. When a salient object shares a similar colour with the background, it is difficult for existing RGB models to extract saliency. With the help of depth information, a salient object can easily be detected by the proposed RGB-D method. In particular, when the salient object shares similar object categories, e.g. the first row in Fig. 10, $\mathrm{MC}$ and MDF generate unsatisfying results without depth cues. ACSD is not designed for such complex scenes but rather single dominant-object depth images. By providing an accurate depth map, the LMH and GP methods perform well in both precision and recall. LMH uses a simple fusion framework that takes advantage of both depth and appearance cues from the low, mid, and high levels. The background is nicely excluded; however, many pixels on the salient object are not detected as salient, e.g. the second row in Fig. 10. Figure 10 also shows that the proposed method consistently outperforms all the other saliency methods.

\subsection{Quantitative evaluation}

Our algorithm is implemented in MATLAB v7.12 and tested on an Intel Core(TM) i5-6400 CPU with 8 GB of RAM. A simple computational comparison is shown in Table 4 in terms of the NLPR dataset without a GPU. The run time of ACSD is for per depth image; GMR, MC and MDF are for per RGB image; and LMH, GP and BFSD are for per RGB-D image pair. Note that there are many works left for computational optimization, including optimization of prior parameters and algorithm optimization for variable inference during the prediction process.

The quantitative comparisons on the NLPR dataset are shown in Figs. 11 and 12. As shown in Fig. 11a, b, although the PR curves are very similar in the colourdepth saliency situation and the colour saliency situation, Fig. 11c shows that the proposed method is superior compared to MC and MDF in the depth saliency situation. The LMH method, which uses Bayesian fusion to fuse depth and RGB saliency by simple multiplication, has lower performance compared to the GP method, which uses the Markov random field model as a fusion strategy, as shown in Fig. 11b, c. LMH and GP achieve better performances than ACSD by using fusion strategies, as shown in Fig. 11.

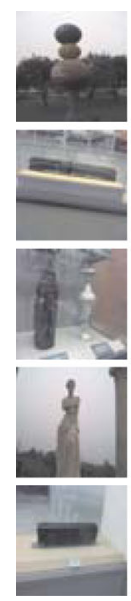

a
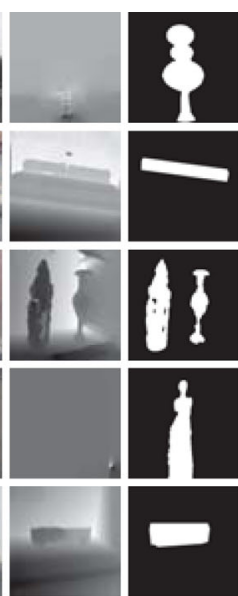

b
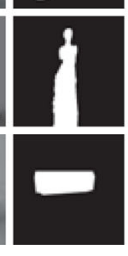

C
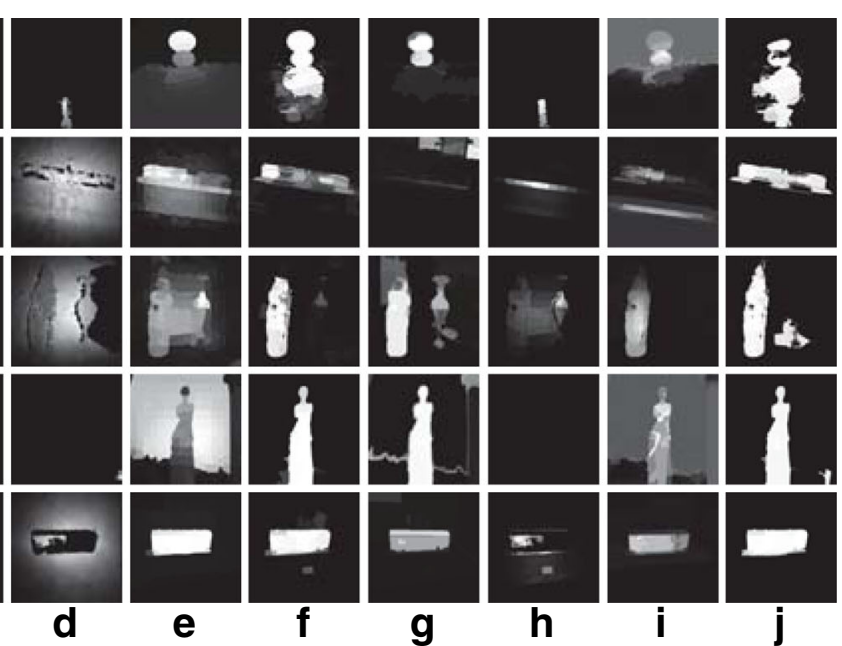

h
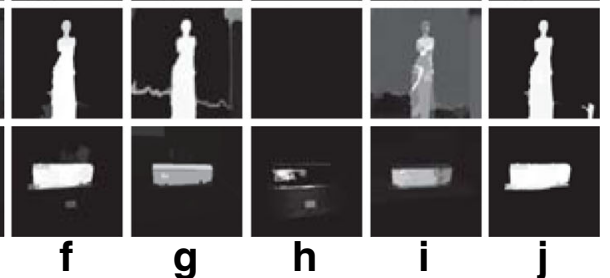

Fig. 9 Visual comparison of the saliency detection in the colour saliency situation in terms of the NLPR dataset. a RGB. b Depth. c Ground truth. d ACSD. e GMR. f MC. g MDF. h LMH. i GP. j BFSD 


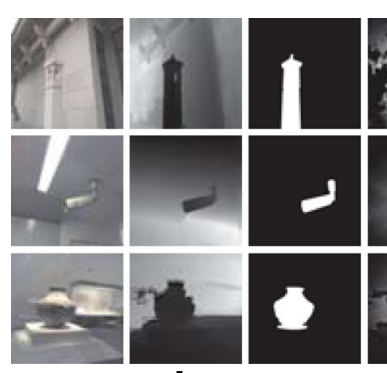

a

b

C

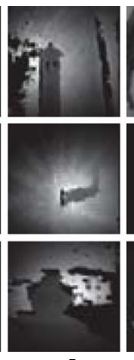

d

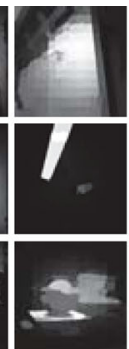

e

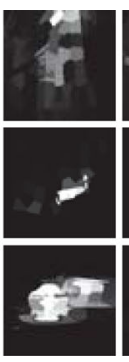

f

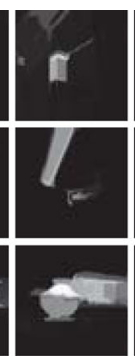

$\mathbf{g}$

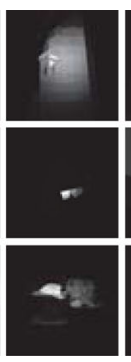

h

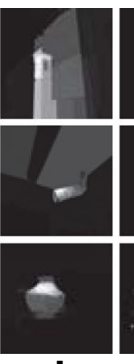

i

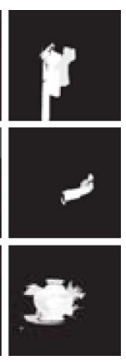

j

Fig. 10 Visual comparison of the saliency detection in the depth saliency situation in terms of the NLPR dataset. a RGB. b Depth. c Ground truth. d ACSD. e GMR. f MC. g MDF. h LMH. i GP. j BFSD

LMH and GP achieve better performances than GMR by using fusion strategies in the depth saliency situation, as shown in Fig. 11c; however, LMH and GP achieve lower performances in the colour saliency situation, as shown in Fig. 11b. The PR curves demonstrate that the proposed 3D saliency detection model performs better than do the compared methods overall, as shown in Fig. 11d. We also provide the F-measure values for several compared methods in Table 5, which shows that the proposed RGB-D method is superior to the existing methods in terms of F-measure values. This result is mainly because the deep features of RGB-D images extracted by CNNs enhance the consistency and compactness of salient patches.

As shown in Fig. 12c, in the depth saliency situation, the RGB saliency methods perform relatively worse than the RGB-D saliency methods in terms of precision. However, in the colour saliency situation, the ACSD and LMH methods do not perform well in both precision and recall. Although the simple late fusion strategy achieves improvements in the depth saliency situation, as shown in Fig. 12c, it still suffers from inconsistency in the homogeneous foreground regions in the colour saliency situation, as shown in Fig. 12b, which may be attributed to treating the appearance and depth correspondence cues in an independent manner. In the colour-depth saliency situation, due to the capability of learning high-level semantic features, MC and MDF perform relatively better than the LMH and GP methods in terms of F-measure. Although the recall values are very similar, Fig. 12b, c show that the proposed method improves the precision and F-measure when compared to MC and MDF. Our approach consistently detects the pixels on the dominant objects within a Bayesian framework with higher accuracy to resolve the

Table 4 Comparison of the average run time (seconds) on the NLPR dataset

\begin{tabular}{llllllll}
\hline Dataset & ACSD & GMR & MC & MDF & LMH & GP & BFSD \\
\hline NLPR & $0.2 \mathrm{~s}$ & $2.9 \mathrm{~s}$ & $72.7 \mathrm{~s}$ & $2.1 \times 10^{3} \mathrm{~s}$ & $2.8 \mathrm{~s}$ & $38.9 \mathrm{~s}$ & $80.1 \mathrm{~s}$ \\
\hline
\end{tabular}

issue. Figure 12 shows that the proposed method performs favourably against the existing algorithm with higher precision, recall values, and F-measure scores on the NLPR dataset.

\subsubsection{Supervision transfer vs fine-tuning}

This section investigates the effectiveness of different depth CNN learning strategies. It was demonstrated that fine-tuning a deep $\mathrm{CNN}$ model for image classification with the target task (e.g. object detection) data can significantly improve the performance of the target task [37]. Supervision transfer enables learning of rich representations from a large labelled modality as a supervisory signal for training representations for a new unlabelled paired modality and can be used as a pre-training procedure for new modalities with limited labelled data. However, the fine-tuning task and the supervision transfer task have disparity in the following aspects. (1) Input data. The fine-tuning task takes the labelled depth images as inputs, while the supervision transfer task requires the paired RGB and depth images. The fine-tuning solve the problem of domain adaptation within the same modality. In contrast, supervision transfer here tackles the problem of domain adaptation across different modalities. (2) The adapted layer. The fine-tuning task adapts the last soft-max layer to the same modality data, while the supervision transfer happens at the arbitrary internal layer for a new image modality. Particularly, deep model structures at the fine-tuning stage are only different in the last fully connected layer for predicting labels. Supervision transfer here allows for transfer of supervision at arbitrary semantic levels. Due to the "data-hungry" nature of CNNs, the existing training data is insufficient for training; therefore, we employed supervision transfer to resolve this issue.

We evaluate the performance of the Depth CNN model with different training strategies on the NLPR dataset. We randomly select 600 depth images $\mathcal{I}_{d}^{b}$ for training and 100 for validation from $\mathcal{D}^{b}$. We show detailed experimental results for supervision transfer from RGB to depth images 

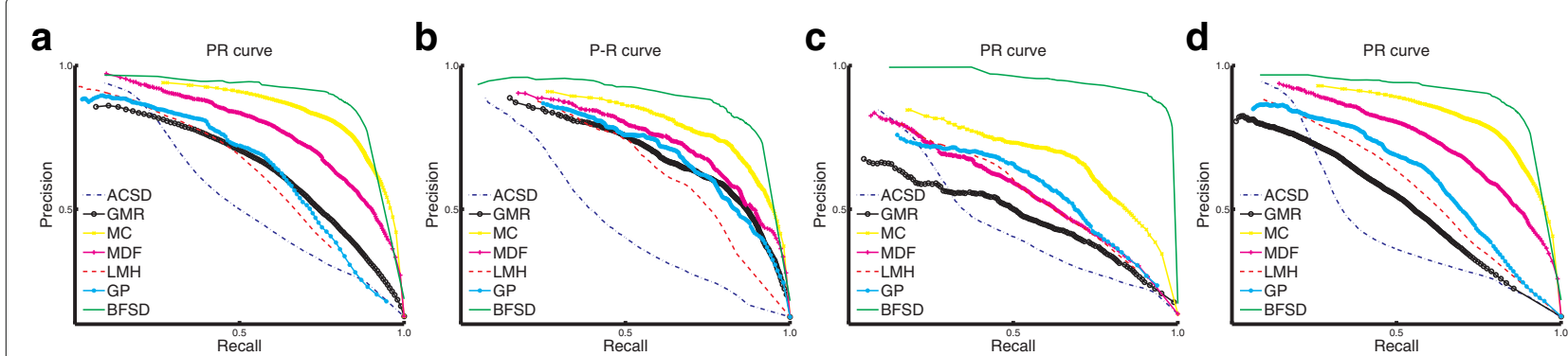

Fig. 11 The PR curves of different saliency detection models in terms of the NLPR dataset. a Colour-depth saliency. b Colour saliency. c Depth saliency. d The overall

compared with fine-tuning with depth images, as shown in Fig. 13. We use the Clarifai that has been trained on labelled images in the ImageNet dataset, and use the midlevel representation learned by the $\mathrm{CNN}$ as a supervisory signal to train a CNN on depth images. Note that the output of the penultimate layer of Depth $\mathrm{CNN}$ is indeed a feature vector for saliency detection. The technique for transferring supervision results in improvements in performance for the end task of saliency detection on NLPR dataset, where we improve from 1.5 to $1.9 \%$ when using both RGB and depth images together, compared with the fine-tuning when using just the depth images. From the results on NLPR dataset in Fig. 13, we can conclude that supervision transfer outperforms the conventional fine-tuning method, which validates the effectiveness of the proposed supervision transfer approach for saliency detection.

\subsubsection{Fusion strategy comparison}

Despite the demonstrated success of deep features extracted from RGB images and Depth images, no single feature is effective for all scenarios as they define saliency from different perspectives. The combination of different features might be a good solution to visual saliency detection for RGB-D images. However, manually designing an interaction mechanism for integrating inherently different saliency features is a challenging problem. The qualitative comparisons and quantitative comparisons of the different fusion strategies using the deep $\mathrm{CNN}$ features are shown in Figs. 14, 15, and 16, respectively. CSM means colour saliency map, which is produced by deep features of the colour CNN. DSM means depth saliency map, which is produced by deep features of the depth CNN. We add and multiple the CSM with the DSM, and these results are denoted CSM+CSM and $\mathrm{CSM} \times \mathrm{DSM}$. As shown in Fig. 14, neither simple linear fusion nor weighting method is subsequently able to recover the salient object. Both simple linear fusion and weighting method suffer from inconsistency in the homogeneous foreground regions and lacks precision around object boundaries, which may be ascribed to treating the colour and depth correspondence cues in an independent manner. Our approach consistently detects the pixels on the dominant objects within a Bayesian framework with higher accuracy to resolve the issue. Figure 15 shows that the Bayesian fusion performs favourably compared with the linear fusion and the weighting method, with higher precision and recall on the NLPR dataset. Although the simple late fusion strategy achieves improvements, it still suffers from inconsistency due to ignore the strong complementarities between appearance and depth correspondence cues. We adopt a good integration method developed to address this problem by training a generative model.

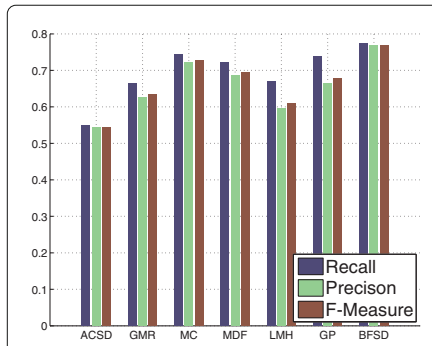

a

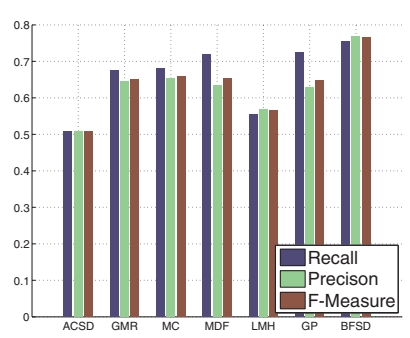

b

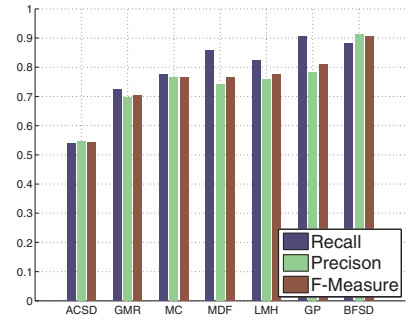

C

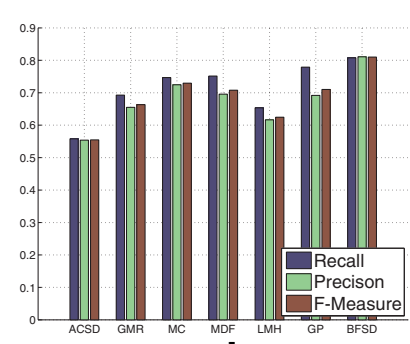

d

Fig. 12 The F-measures of different saliency detection models when used on the NLPR dataset. a Colour-depth saliency. b Colour saliency. c Depth saliency. d The overall 
Table 5 Comparison of the F-measure on the NLPR dataset

\begin{tabular}{|c|c|c|c|c|c|c|c|}
\hline 3D saliency situation & ACSD & GMR & $M C$ & MDF & $\mathrm{LMH}$ & GP & BFSD \\
\hline Colour-depth saliency & 0.5548 & 0.6540 & 0.7381 & 0.6983 & 0.6109 & 0.6891 & 0.7793 \\
\hline Colour saliency & 0.5195 & 0.6612 & 0.6684 & 0.6630 & 0.5645 & 0.6480 & 0.7658 \\
\hline Depth saliency & 0.5635 & 0.7032 & 0.7711 & 0.7689 & 0.7744 & 0.8095 & 0.9044 \\
\hline Overall & 0.5510 & 0.6652 & 0.7366 & 0.7058 & 0.6317 & 0.7082 & 0.8092 \\
\hline
\end{tabular}

The best results are shown in Italics

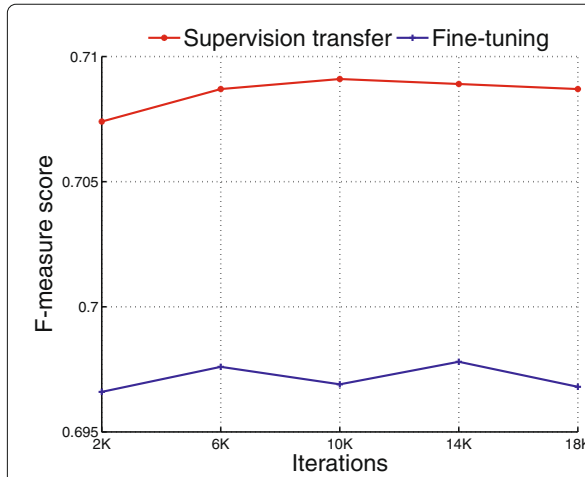

a

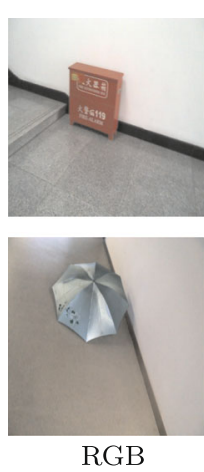

b

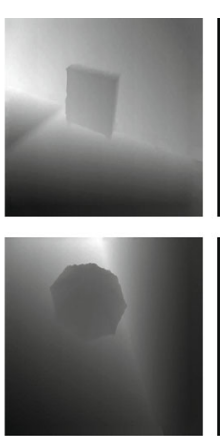

Depth

C
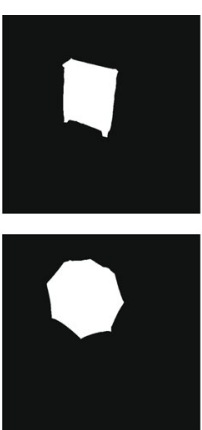

GT

d
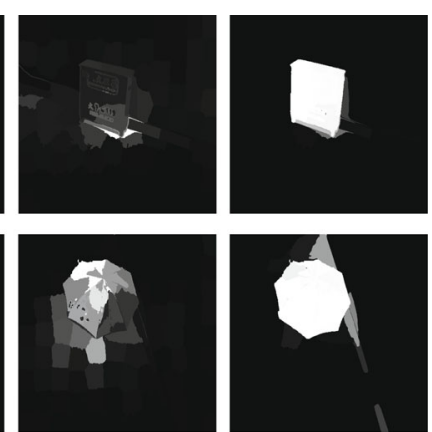

FT

e

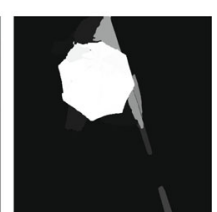

ST

f

Fig. 13 Evaluation of the depth CNN learned using different training strategies on the NLPR dataset. a F-measure scores on the NLPR dataset for evaluation of the supervision transfer (ST) strategy and the fine-tuning (FT) strategy. b-f Qualitative comparison between the ST strategy and the FT strategy for $10 \mathrm{k}$ iterations
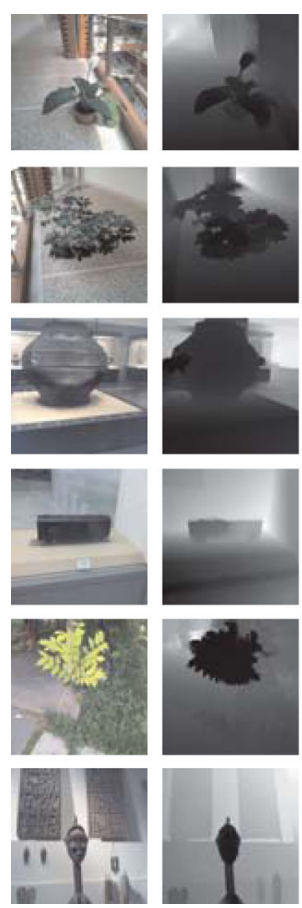

a

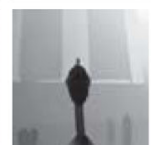

b
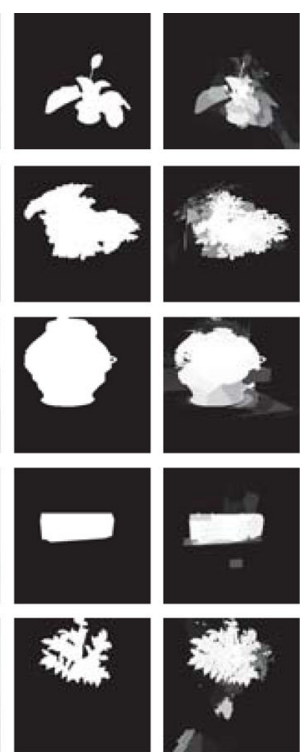

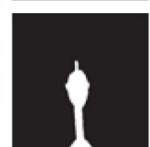

c
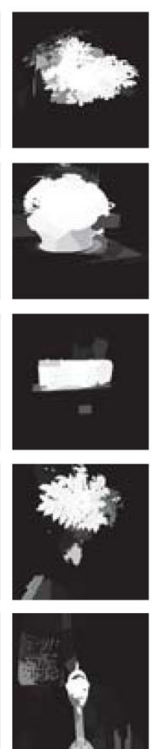

d
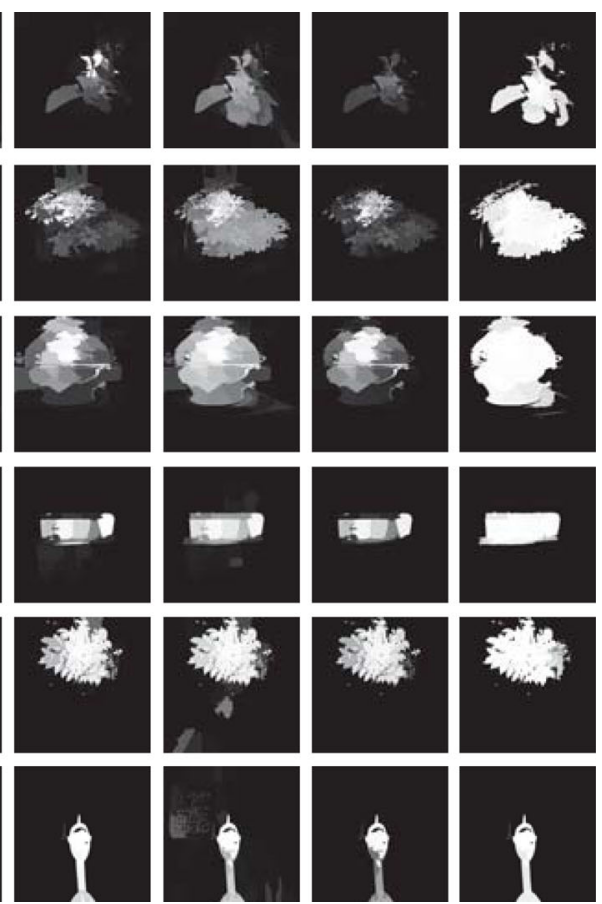

e
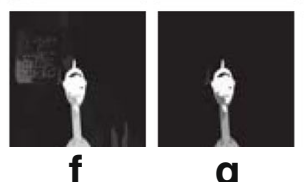

g

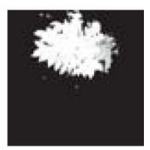

Fig. 14 Visual comparison of different fusion strategies in terms of the NLPR dataset. a RGB. b Depth. c Ground truth. d CSM. e DSM. f CSM + DSM. $\mathbf{g}$ $\mathrm{CSM} \times \mathrm{DSM}$. $\mathbf{h}$ BFSD. The symbol " + " indicates a linear combination strategy, and the symbol " $\mathrm{x}$ " indicates a weighting method based on multiplication 

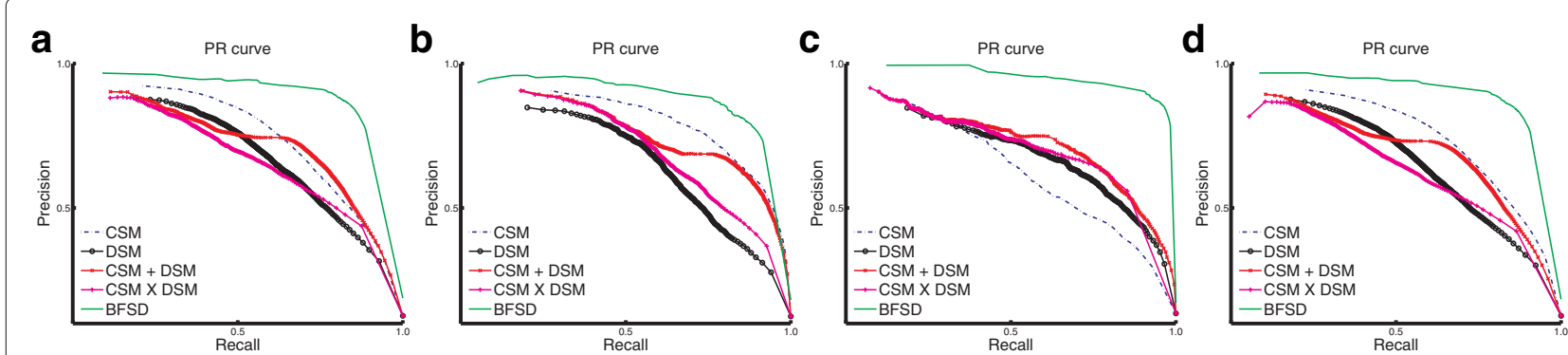

Fig. 15 The PR curves of different fusion strategies in terms of the NLPR dataset. a Colour-depth saliency. b Colour saliency. c Depth saliency. d The overall. The symbol " + " indicates a linear combination strategy, and the symbol " $x$ " indicates a weighting method based on multiplication

\subsection{Cross-dataset generalization}

In this section, we evaluate the generalization performance of BFSD. To test how well the performance of our proposed method generalizes to a different dataset for detecting salient object in RGB-D images, we evaluate it on the NJU-DS400[10]. As discussed in experiment setting, the images of the NJU-DS400 are collected in different scenarios. We directly test the performance on the NJU-DS400 dataset with the model learned on NLPR dataset. The results are shown in Figs. 17 and 18. In the NJU-DS400 dataset, we do not have experimental results for the LMH and GP methods due to the lack of depth information, which is required by their codes. Although the model is trained on the NLPR dataset, it outperforms all other previous methods based on the F-measure scores and PR curves. This clearly demonstrates the generalization performance of our proposed method and robustness to dataset biases. Our model explores high-level information of RGB-D images to investigate semantics-driven attention to $3 \mathrm{D}$ content, and has much stronger generalization capability. Though Gaussian distributions of the DMNB model provide better performance than compared methods in terms of NJU-DS400 dataset, different numbers of mixture component would impair the generalization capability of this mixture model, especially in the case of multiple scene types.

\subsubsection{Failure cases}

Figure 19 presents more visual results and some failure cases of our proposed method on NLPR dataset. By comparing these images, we find that semantic information is more helpful when the salient object shares a very similar colour and depth information with the background. Figure 20 presents additional visual results and a failure case of our proposed method on NJU-DS400 dataset. We find that although our method is able to highlight the overall salient objects, the generated coarse maps may confuse some of small foreground or background regions if they have similar appearance. Our method may fail when the salient object shares a very similar colour and depth information with the background in a global context.

\subsubsection{Limitations}

Because our approach requires training on large datasets to adapt to specific environments, it has the problem that properly tuning the parameters for specific new tasks is important to the performance of the DMNB model. The DMNB model performs classification in one shot via a

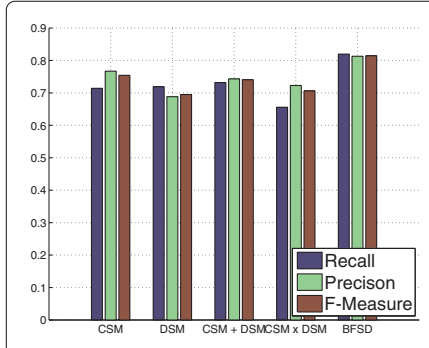

a

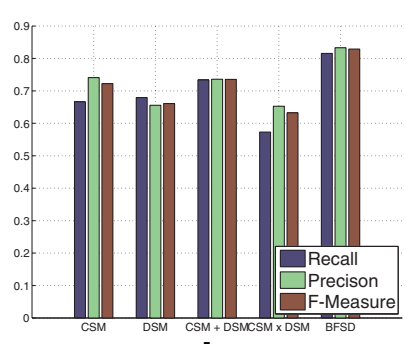

b

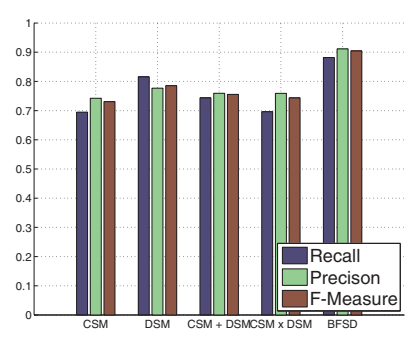

C

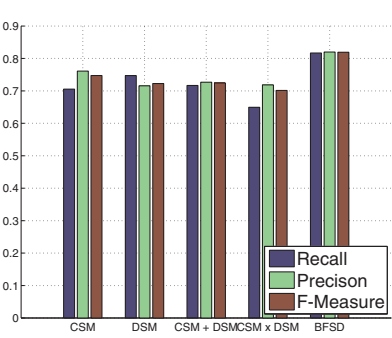

d

Fig. 16 The F-measure scores of different fusion strategies in terms of the NLPR dataset. a Colour-depth saliency. b Colour saliency. c Depth saliency. d The overall. The symbol " + " indicates a linear combination strategy, and the symbol " $x$ " indicates a weighting method based on multiplication 


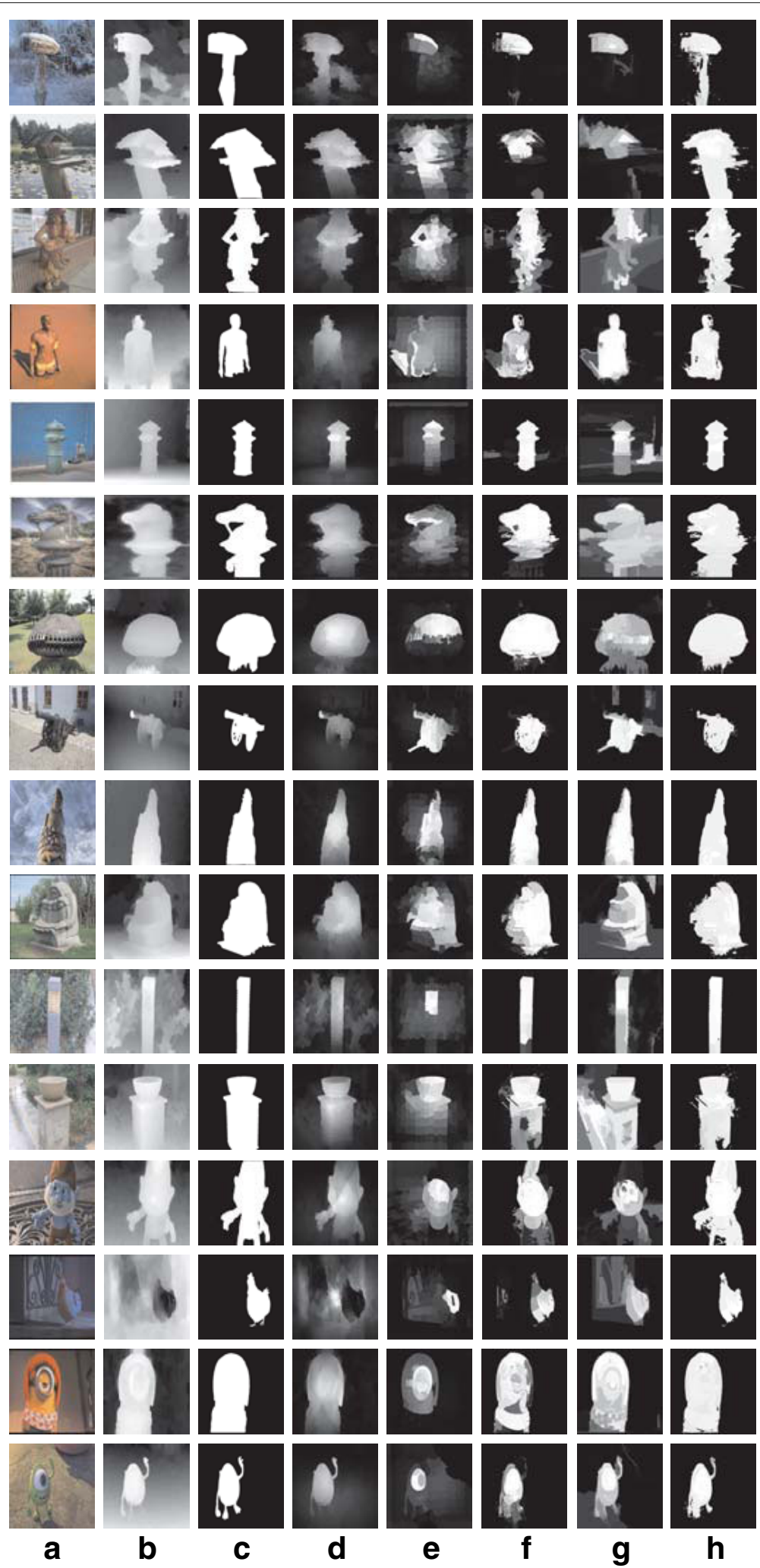

Fig. 17 Visual comparison of the saliency detection in terms of the NJU-DS400 dataset. a RGB. b Depth. c Ground truth. d ACSD. e GMR. f MC. $\mathbf{g}$ MDF. h BFSD 
a

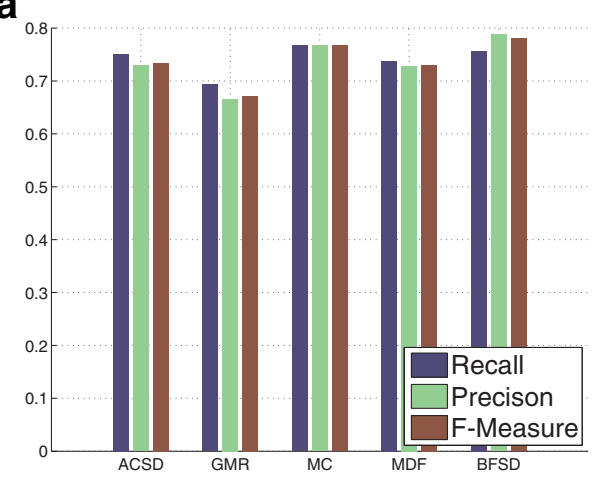

b

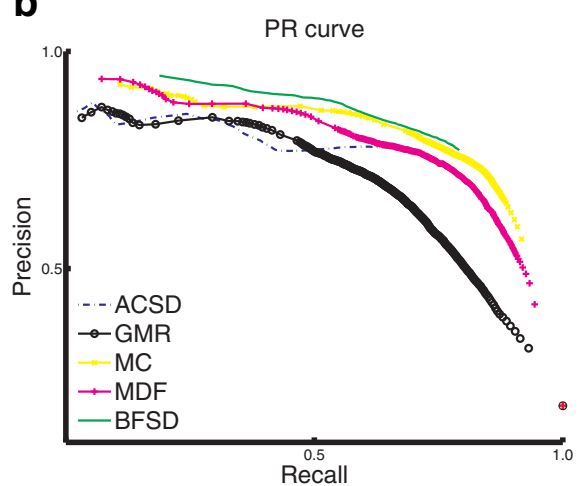

Fig. 18 Experimental results on the NJU-DS400 dataset compared with previous works. a The F-measure scores. b The PR curves. F-measure scores and PR curves show superior generalization ability of a BFSD framework

combination of mixed-membership models and logistic regression, where the results may depend on different choices of $C$. The learned parameters will clearly have good performances on the specific stimuli but not necessarily on the new testing set. Thus, the weakness of the proposed method is that to obtain reasonable performances, we train our saliency model on the training set for specific $C$. This problem could be addressed by using Dirichlet process mixture models to find a proper $C$ for new datasets.

\section{Conclusion}

In this study, we propose a learning-based 3D saliency detection model for RGB-D images that considers the deep features of RGB images and depth images within a Bayesian framework. To better detect semantically salient objects, we employ a deep CNN to model saliency of objects in RGB images and depth images. Rather than simply combining a depth map with 2D saliency maps as in previous studies, we propose a computational saliency detection model for RGB-D images based on the DMNB model. The experiments verify that the deep features of depth images can serve as a helpful complement to the deep features of RGB images within a Bayesian framework. Compared with other competing 3D models, the experimental results from a public RGB-D saliency datasets demonstrate the improved performance of the proposed model over other strategies.

As a future work, we are considering to improve the feature representation of the depth images. We are considering to represent the depth image by three

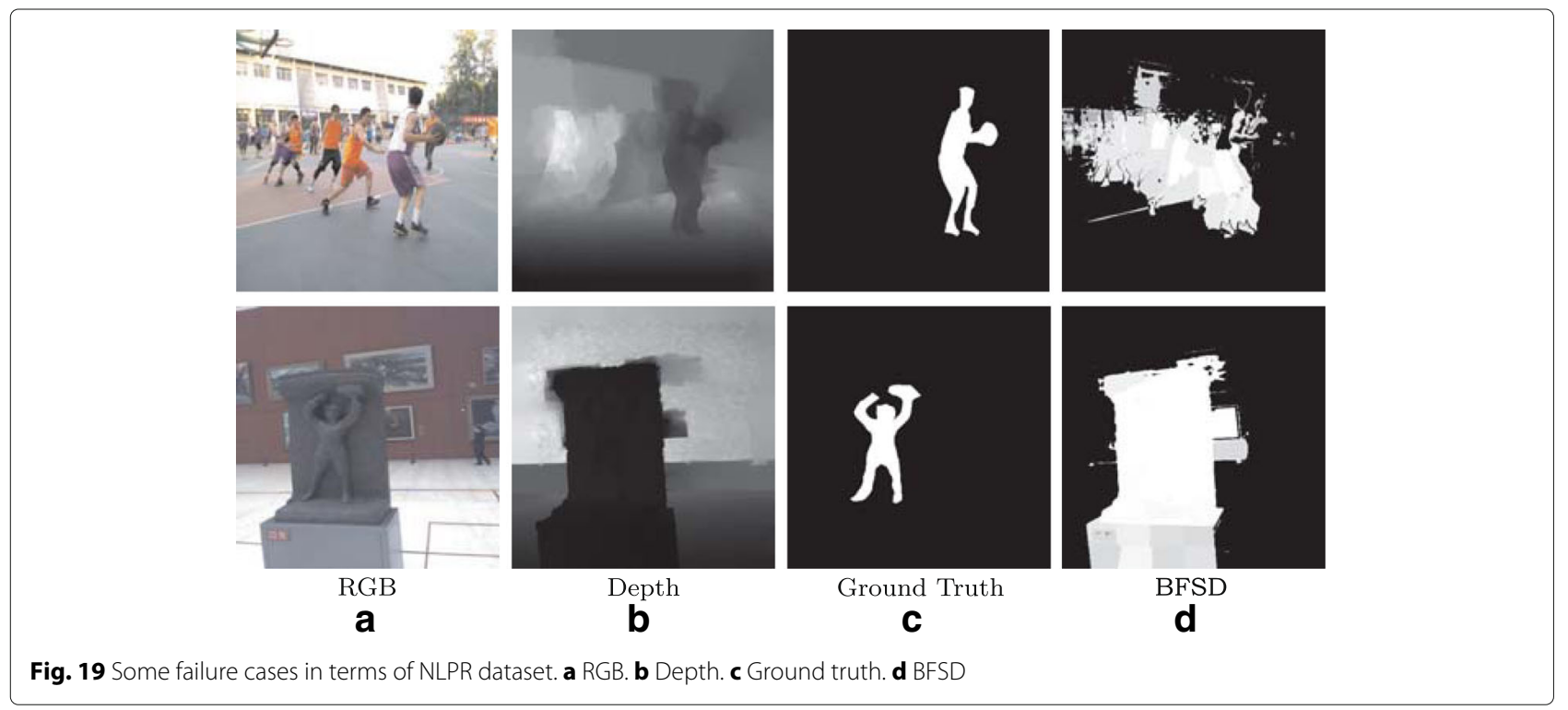



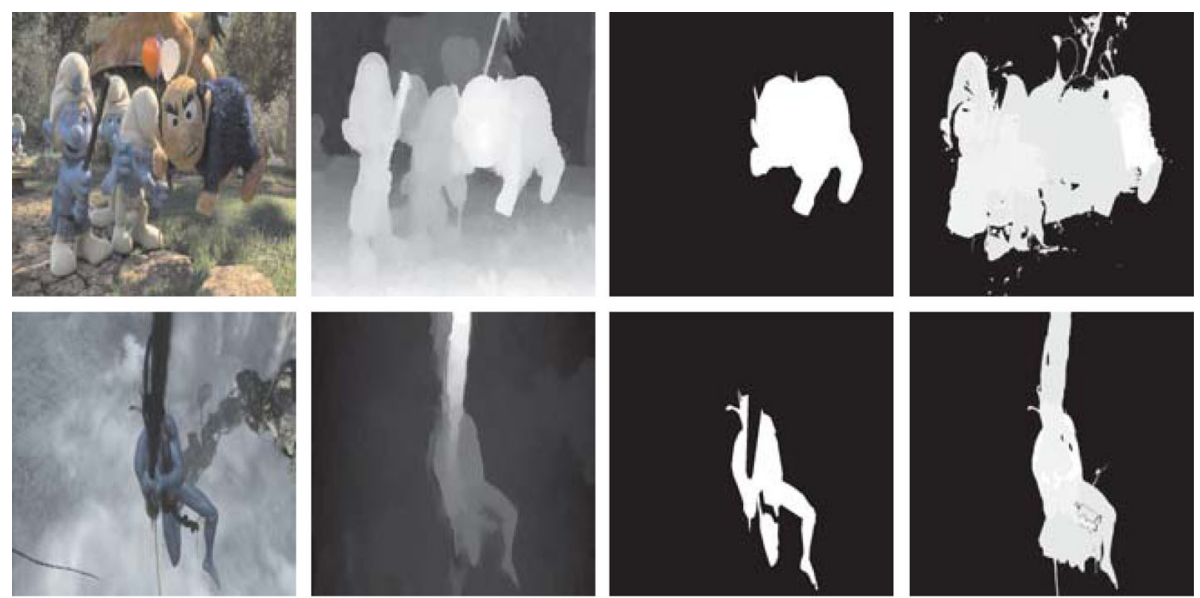

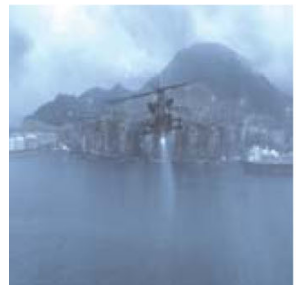

RGB

a

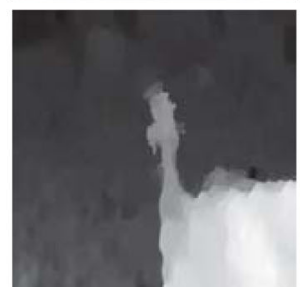

Depth

b

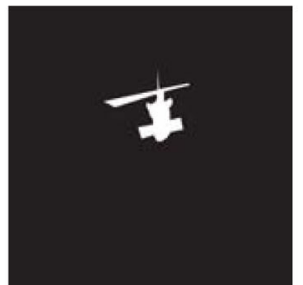

Ground Truth

C

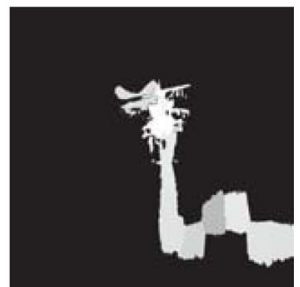

BFSD

d

Fig. 20 Some failure casesin terms of NJU-DS400 dataset. a RGB. b Depth. c Ground truth. d BFSD

channels (horizontal disparity, height above ground, and angle with gravity) [38] for saliency detection because this representation allows the $\mathrm{CNN}$ to learn stronger features than by using disparity alone. We are also considering the application of our 3D saliency detection model in RGB-D object detection problems, e.g. 3D object proposals.

\section{Endnotes}

${ }^{1} \mathrm{http}: / /$ sites.google.com/site/rgbdsaliency

${ }^{2}$ http://mcg.nju.edu.cn/en/resource.html

\section{Acknowledgements}

This work was supported in part by the Beijing Municipal special financial project (PXM2016_278215_000013, ZLXM_2017C010) and by the Innovation Group Plan of Beijing Academy of Science and Technology (IG201506C2).

\section{Authors' contributions}

SW took charge of the system coding, doing experiments, data analysis and writing the whole paper excluding variational EM algorithm part at subsection 3.3. ZZ took charge of advisor position for paper presentation and experiment design. WJ took charge of data analysis presentation as well as English revising. $\mathrm{HQ}$ took charge of coding and writing for variational EM algorithm part at subsection 3.3. All authors read and approved the final manuscript.

\section{Competing interests}

The authors declare that they have no competing interests.

\section{Publisher's Note}

Springer Nature remains neutral with regard to jurisdictional claims in published maps and institutional affiliations.

\section{Author details}

${ }^{1}$ The Higher Educational Key Laboratory for Measuring and Control Technology and Instrumentations of Heilongjiang Province, Harbin University of Science and Technology, Harbin, 150080 China. ${ }^{2}$ Res. Center for Artif. Intell. and Big Data Anal., Beijing Academy of Science and Technology, Beijing, 100094 China.

Received: 31 May 2017 Accepted: 13 December 2017

Published online: 10 January 2018

\section{References}

1. Le Callet P, Niebur E (2013) Visual attention and applications in multimedia technology. Proc IEEE 101(9):2058-2067. https://doi.org/10. 1109/JPROC.2013.2265801

2. Wang J, Fang Y, Narwaria M, Lin W, Callet PL (2014) Stereoscopic image retargeting based on $3 \mathrm{~d}$ saliency detection. In: The IEEE International Conference on Acoustics, Speech and Signal Processing. IEEE, Florence. pp 669-673

3. Kim H, Lee S, Bovik C (2014) Saliency prediction on stereoscopic videos. IEEE Trans Image Process 23(4):1476-1490. https://doi.org/10.1109/TIP. 2014.2303640

4. Zhang Y, Jiang G, Yu M, Chen K (2010) Stereoscopic visual attention model for 3d video. In: The 16th International Conference on Multimedia Modeling. Springer, Chongqing. pp 314-324

5. Borji A, Cheng M, Hou Q, Jiang H, Li J (2017) Salient object detection: a survey. arXiv preprint arXiv:1411.5878

6. Borji A, Cheng M, Jiang H, Li J (2015) Salient object detection: a benchmark. IEEE Trans Image Process 24(12):5706-5722. https://doi.org/ 10.1109/TIP.2015.2487833

7. Yang C, Zhang L, Lu H, Ruan X, Yang M (2013) Saliency detection via graph based manifold ranking. In: The IEEE Conference on Computer Vision and Pattern Recognition. IEEE, Portland. pp 3166-3173

8. Peng H, Li B, Ling H, Hu W, Xiong W, Maybank SJ (2017) Salient object detection via structured matrix decomposition. IEEE Trans Pattern Anal Mach Intell 39(4):818-832. https://doi.org/10.1109/TPAMI.2016.2562626 
9. Li B, Xiong W, Hu W (2012) Visual saliency map from tensor analysis. In: Proceedings of Twenty-Sixth AAAI Conference on Artificial Intelligence. AAAl, Toronto. pp 1585-1591

10. Ju R, Ge L, Geng W, Ren T, Wu G (2014) Depth saliency based on anisotropic centre-surround difference. In: IEEE International Conference Image Processing. IEEE, Pairs. pp 1115-1119

11. Song H, Liu Z, Xie Y, Wu L, Huang M (2016) Rgbd co-saliency detection via bagging-based clustering. IEEE Sig Process Lett 23(12):1722-1726. https://doi.org/10.1109/LSP.2016.2615293

12. Lang C, Ngugen T, Katti H, Yadati K, Kankanhalli M, Yan S (2012) Depth matters: influence of depth cues on visual saliency. In: The 12th European Conference Computer Vision. Springer, Florence. pp 101-105

13. Desingh K, Madhava K, Rajan D, Jawahar C (2013) Depth really matters: improving visual salient region detection with depth. In: The British Machine Vision Conference. BMVA, Bristol. pp 98.1-98.11

14. Shan H, Banerjee A, Oza N (2009) Discriminative mixed-membership models. In: IEEE International Conference Data Mining. IEEE, Miami. pp 466-475

15. Fang $Y$, Wang J, Narwaria M, Le Callet P, Lin W (2014) Saliency detection for stereoscopic images. IEEE Trans Image Process 23(6):2625-2636. https://doi.org/10.1109/TIP.2014.2305100

16. Wu P, Duan L, Kong L (2015) Rgb-d salient object detection via feature fusion and multi-scale enhancement. In: Chinese Conference Computer Vision. Springer, Xi'an. pp 359-368

17. Ciptadi A, Hermans T, Rehg J (2013) An in depth view of saliency. In: The British Machine Vision Conference. BMVA, Bristol. pp 9-13

18. latsun I, Larabi M, Fernandez-Maloigne C (2014) Using monocular depth cues for modeling stereoscopic 3D saliency. In: IEEE International Conference Acoustics, Speech and Signal Processing. IEEE, Florence. pp 589-593

19. Peng H, Li B, Hu W, Ji R (2014) Rgbd salient object detection: a benchmark and algorithms. In: The 13th European Conference Computer Vision. Springer, Zurich. pp 92-109

20. Ren J, Gong X, Yu L, Zhou W (2015) Exploiting global priors for rgb-d saliency detection. In: IEEE Conference Computer Vision and Pattern Recognition Workshops. IEEE, Boston. pp 25-32

21. Xue H, Gu Y, Li Y, Yang J (2015) Rgb-d saliency detection via mutual guided manifold ranking. In: IEEE International Conference Image Processing. IEEE, Quebec. pp 666-670

22. Wang J, DaSilva M, Le Callet P, Ricordel V (2013) Computational model of stereoscopic 3D visual saliency. IEEE Trans Image Process 22(6):2151-2165. https://doi.org/10.1109/TIP.2013.2246176

23. Fang Y, Lin W, Fang Z, Lei J, Le Callet P, Yuan F (2014) Learning visual saliency for stereoscopic images. In: IEEE International Conference Multimedia and Expo Workshops. IEEE, Chengdu. pp 1-6

24. Zhu L, Cao Z, Fang Z, Xiao Y, Wu J, Deng H, Liu J (2015) Selective features for RGB-D saliency. In: Conference Chinese Automation Congress. IEEE, Wuhan. pp 512-517

25. Bertasius G, Park H, Shi J (2015) Exploiting egocentric object prior for 3d saliency detection. arXiv preprint arXiv:1511.02682

26. Qu L, He S, Zhang J, Tian J, Tang Y, Yang Q (2013) RGBD salient object detection via deep fusion. IEEE Trans Image Process 26(5):2274-2285. https://doi.org/10.1109/TIP.2017.2682981

27. Zhao R, Ouyang W, Li H, Wang X (2015) Saliency detection by multi-context deep learning. In: IEEE Conference Computer Vision and Pattern Recognition Workshops. IEEE, Boston. pp 1265-1274

28. Li G, Yu Y (2016) Visual saliency detection based on multiscale deep CNN features. arXiv preprint arXiv:1609.02077

29. Zeiler M, Fergus R (2014) Visualizing and understanding convolutional networks. In: The 13th European Conference Computer Vision. Springer Zurich. pp 818-833

30. Gupta S, Hoffman J, Malik J (2015) Cross modal distillation for supervision transfer. arXiv preprint arXiv:1507.00448

31. Wang S, Zhou Z, Qu H, Li B (2016) Visual saliency detection for RGB-D images with generative model. In: The 13th Asian Conference on Computer Vision. Springer, Taipei. pp 20-35

32. Rish I (2001) An empirical study of the naive Bayes classifier. J Univ Comput Sci 3(22):41-46

33. Blei $D$, Jordan $M(2006)$ Variational inference for dirichlet process mixtures. Bayesian Anal 1(1):121-143
34. Jia Y (2013) Caffe: An open source convolutional arichitecture for fast feature embedding. http://caffe.berkeleyvision.org/. Accessed 2013

35. Russakovsky O, Deng J, Su H, Krause J, Satheesh S, Ma S, Huang Z, Karpathy A, Khosla A, Bernstein M, Berg AC, Li F (2014) Imagenet large scale visual recognition challenge. Int J Comput Vis 115(3):211-252

36. Cheng M, Mitra N, Huang X, Torr P, Hu S (2014) Global contrast based on salient region detection. IEEE Trans Image Process 37(3):569-582. https:// doi.org/10.1109/TPAMI.2014.2345401

37. Girshick R, Donahue J, Darrell T, Malik J (2013) Rich feature hierarchies for accurate object detection and semantic segmentation. arXiv preprint arXiv:1311.2524

38. Gupta S, Girshick R, Arbelaez P, Malik J (2014) Learning rich features from RGB-D images for object detection and segmentation. In: The 13th European Conference Computer Vision. Springer, Zurich. pp 345-360

\section{Submit your manuscript to a SpringerOpen ${ }^{\circ}$ journal and benefit from:}

- Convenient online submission

- Rigorous peer review

- Open access: articles freely available online

- High visibility within the field

- Retaining the copyright to your article

Submit your next manuscript at springeropen.com 